\title{
Ship Pipe Routing Design Using NSGA-II and Coevolutionary Algorithm
}

\author{
Wentie Niu, Haiteng Sui, Yaxiao Niu, Kunhai Cai, and Weiguo Gao \\ Key Laboratory of Mechanism Theory and Equipment Design of Ministry of Education, Tianjin University, Tianjin 300072, China \\ Correspondence should be addressed to Wentie Niu; niuwentie@tju.edu.cn
}

Received 9 July 2016; Revised 6 November 2016; Accepted 30 November 2016

Academic Editor: Francisco Chicano

Copyright (c) 2016 Wentie Niu et al. This is an open access article distributed under the Creative Commons Attribution License, which permits unrestricted use, distribution, and reproduction in any medium, provided the original work is properly cited.

\begin{abstract}
Pipe route design plays a prominent role in ship design. Due to the complex configuration in layout space with numerous pipelines, diverse design constraints, and obstacles, it is a complicated and time-consuming process to obtain the optimal route of ship pipes. In this article, an optimized design method for branch pipe routing is proposed to improve design efficiency and to reduce human errors. By simplifying equipment and ship hull models and dividing workspace into three-dimensional grid cells, the mathematic model of layout space is constructed. Based on the proposed concept of pipe grading method, the optimization model of pipe routing is established. Then an optimization procedure is presented to deal with pipe route planning problem by combining maze algorithm (MA), nondominated sorting genetic algorithm II (NSGA-II), and cooperative coevolutionary nondominated sorting genetic algorithm II (CCNSGA-II). To improve the performance in genetic algorithm procedure, a fixed-length encoding method is presented based on improved maze algorithm and adaptive region strategy. Fuzzy set theory is employed to extract the best compromise pipeline from Pareto optimal solutions. Simulation test of branch pipe and design optimization of a fuel piping system were carried out to illustrate the design optimization procedure in detail and to verify the feasibility and effectiveness of the proposed methodology.
\end{abstract}

\section{Introduction}

Since the 1970s, pipe routing design has been studied in various industrial fields, like aeroengine, large-scale integrated circuit, ship, and so forth. Pipe routing design, which is related to other tasks, is one of the most important processes at the detailed design stage of a ship. However, duo to the complexity of piping system and the diversity of constraints in ship piping system, it is time-consuming and difficult to achieve feasible layout. Therefore, it is significant to investigate automatic pipe routing method.

Systematic studies in route path planning have been carried out by researchers for several decades. Dijkstra algorithm [1] proposed in 1959 is a well-known algorithm for path optimization with shortest length. Based on Dijkstra algorithm, $A^{*}$ algorithm is developed by Hart et al. [2] to improve search efficiency. In 1961, Lee [3] proposed maze algorithm to solve connecting problem of two points. However, huge data storage is essential to handle large-size optimization problems, which results in low search efficiency.
To overcome the mentioned drawbacks, numerous researches have been conducted in references [4-8]. Typically, the escape algorithm is proposed by Hightower [4] to improve search efficiency, but an optimal solution also cannot be guaranteed. Recently, researches on piping route path planning have been promoted by the development of modern optimization algorithms such as genetic algorithm [9-17], ant colony algorithm [18-23], and particle swarm optimization [24-27]. Genetic algorithm is used by Ito [10] to optimize pipe routing in two-dimensional space, in which the chromosome of route path with variable-length is firstly defined. Afterward, the chromosome with fixed length [12] is redefined to solve the problems such as low efficiency in GA. A variable-length encoding technique is proposed by Fan et al. [13] and applied to optimize the ship pipe paths in three-dimensional space, which leads to the complexity of algorithm design process. In addition, expert systems [28-31] are also applied in ship pipe route design.

At present, the optimization algorithm research on pipe route planning mainly concentrates on the case with two 
terminals, while multibranch pipe design is rarely studied. Park and Storch [29] developed a cell-generation method for pipe routing in a ship engine room, in which the branch pipeline is regarded as a compound of two simple forms: endforked and middle-forked. Using maze algorithm and Steiner tree theory, Fan et al. [7] conduct the research on pipe routing problem of aeroengine. Coevolutionary algorithm is applied by $\mathrm{Wu}$ et al. [32] for multibranch pipe routing in ship. Steiner minimal tree and particle swarm optimization are combined by Liu and Wang [33] to solve the optimization problem of multibranch pipe routing in aeroengine. However, fewer researchers have taken the differences of pipeline diameters into consideration. When pulsating pressure is existing in pipeline, the excitation force will be generated at the branch point because of the big difference in diameter value of the connected pipes, which further influences working period and usage security of pipeline [34]. Therefore, the diameter differences should be taken into consideration in optimization algorithm.

Owing to the diameter differences of branch pipelines, the concept of pipe grading is defined in this paper. In consideration of the number differences of connecting points in each grade, a new algorithm is proposed by combining maze algorithm (MA) [3], nondominated sorting genetic algorithm II (NSGA-II) [35], and cooperative coevolutionary nondominated sorting genetic algorithm II (CCNSGA-II) [36]. To improve the performance in genetic algorithm procedure, a fixed-length encoding method is presented based on the improved maze algorithm and adaptive region strategy. In addition, fuzzy set theory is employed to extract the best compromise pipeline from Pareto optimal solutions, by which the imprecise nature of decision maker's judgment is avoided.

The rest of this paper is organized as follows. The problem of ship pipe route design is formulated in Section 2. The proposed pipe routing algorithm is presented in Section 3. A case study is conducted to verify the proposed algorithm in Section 4. Finally, conclusions are drawn in Section 5.

\section{Problem Formulation}

2.1. Representation of Piping Layout Space. Due to the complex ship hull structure and diverse equipment with various shapes, it is time-consuming and inefficient to describe all geometric information in detail for pipe routing design in ship piping layout space. Therefore, it is essential to simplify the environment of ship piping layout. To construct a reasonable workspace model representing the essential geometric information of the equipment and ship hull structure, several principles should be obeyed in the environment simplification of piping layout.

(1) The geometrical properties of the model should be simple;

(2) Spatial position of the model should be expressed accurately;

(3) The accurate spatial positions of the pipeline terminals should be guaranteed.
2.1.1. Simplification of Equipment Model. Informationally complete models are proposed in literature [37] for design constraints based on an analysis of geometric and nongeometric properties of the related space volumes, which is suitable for component layout problem. For the problem of piping system layout, since the equipment locations have already been determined, the simplification of components is just required, and the simplest approach is to establish axis aligned bounding box (AABB). However, if the equipment model is complex, it is difficult for $\mathrm{AABB}$ to meet the requirements for accurate expression of spatial location. As shown in Figure 1, the equipment model simplified by AABB is illustrated in Figure 1(b), in which the model information of original equipment in Figure 1(a) is not described accurately. In this paper, the optimized subdivision boundary box method described in literature [38] is introduced to simplify the models of ship equipment, an example of simplification is shown in Figure 1(c), and other equipment involved in layout space are simplified by the same way. The procedures of optimized subdivision boundary box method are as follows.

Step 1. Construct the AABB of the equipment model.

Step 2. Divide AABB by using the nonuniform grid of cells according to the characteristics of the equipment.

Step 3. For each cell, find the polygons of the model that lie inside or intersect with it.

Step 4. Construct the AABB of the polygons by Step 3.

Step 5. Clip the AABB with the cell itself.

Step 6. Loop to Step 3 until all cells in the grid are processed.

2.1.2. Mathematical Model of Workspace. To represent the geometric information of ship hull structure, components, and pipeline terminals of layout space, the space division is necessary. The irregular workspace is approximately represented by being divided into numbers of cube grids, and the detailed processes are as follows.

The working space is considered as a cuboid space divided into $M \times N \times L$ uniform 3D cubic grid cells, and an example is shown in Figure 2(a). To build the mathematical model of layout space, one vertex cell of the cuboid should be selected as the origin whose coordinate is $(1,1,1)$ to establish a coordinate system, and each cell will be given a specific coordinate $(x, y, z)$ based on the row, column, and floor in the space. The default tag value of each cell is set as " 0 " representing the feasible search space. The cell occupied by obstacles is set as "\#" representing the infeasible search space. Since the obstacles mainly include ship hull, spaces outside the hull, equipment, and the generated pipelines can be described by the diagonal coordinates of one or more cuboids. Grid cells occupied partially by cuboids are approximately regarded as being occupied completely. Figure 2(b) shows an example of approximate representation for part of the simplified ship hull. 


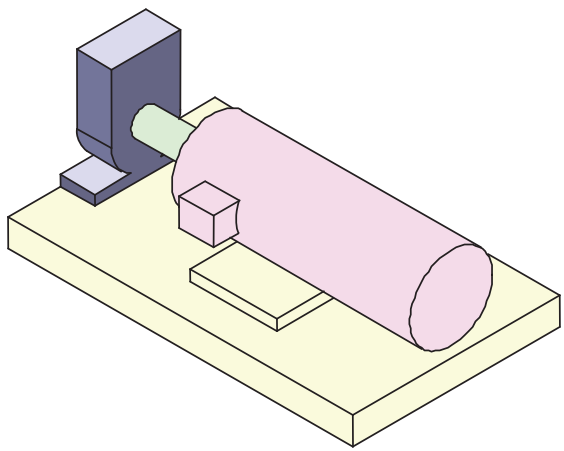

(a)

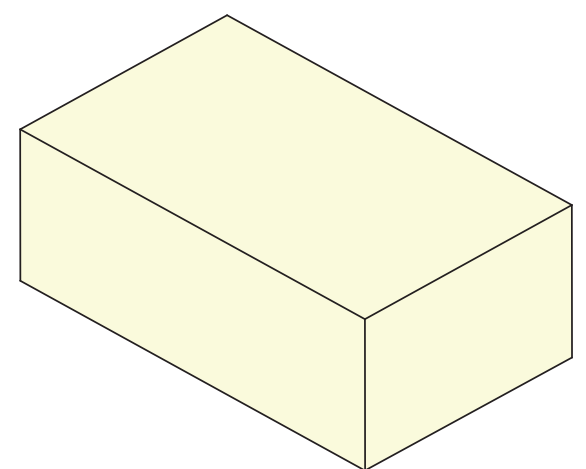

(b)

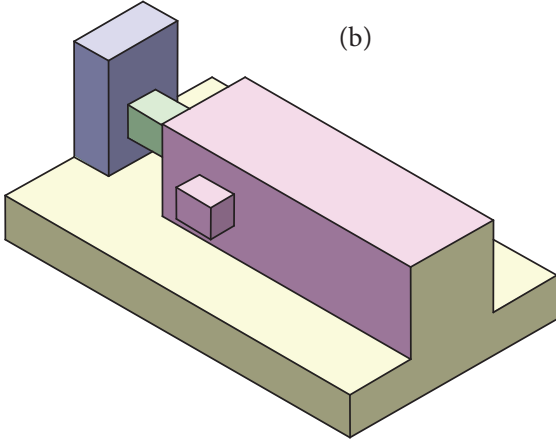

(c)

Figure 1: An example for simplification of the equipment model.

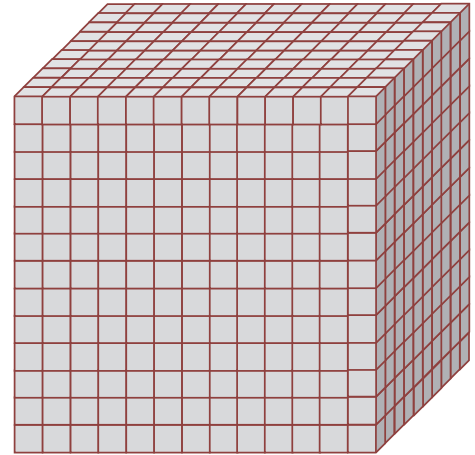

(a)

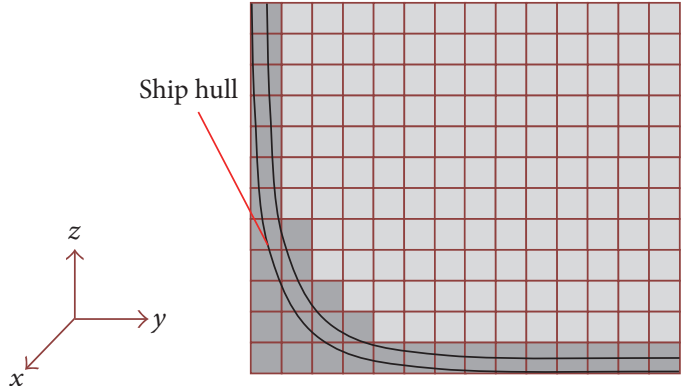

Cells on obstacle

(b)

FIgURE 2: Mathematical model of workspace. (a) Grid method. (b) An example of approximate representation for the simplified ship hull.

\subsection{Description of Pipe Information}

2.2.1. Redefinition of Connecting Point. Since the inlet/outlet of equipment is involved in the simplified model, the equipment simplification will result in failed connection of the pipeline terminals in model space. To solve this problem, the actual inlet/outlet involved in one or several grids is extended to the adjacent grids outside the simplified model along its normal. And the adjacent grid cell passed by the axis of inlet/outlet is defined as new connecting point.

2.2.2. Definition of Pipe Path. Base on the above spacedividing method, a connected pipe path is defined as one continuous path connecting a starting point and a goal point, which contains a series of adjacent grid cells. The encoding of path is represented by a sequence containing a series of coordinates of the grid cells, and an example is shown in Figure 3. The coordinate system is established after the layout space is meshed, in which a green pipe path connecting two object points is generated by taking blue grid cell 1 as a starting point and blue grid cell 2 as the goal point. Apparently, coordinates of grid cells 1 and 2 are, respectively, $(1,2,5)$ and $(9,7,1)$, and the illustrated path code is $\left\{\left(\begin{array}{lll}1 & 2 & 5\end{array}\right),\left(\begin{array}{lll}1 & 3 & 5\end{array}\right),\left(\begin{array}{lll}1 & 4 & 5\end{array}\right),\left(\begin{array}{lll}1 & 5 & 5\end{array}\right),\left(\begin{array}{lll}1 & 6 & 5\end{array}\right),\left(\begin{array}{lll}1 & 7 & 5\end{array}\right)\right.$, (1 $\left.7 \begin{array}{ll}1 & 4\end{array}\right),\left(\begin{array}{lll}1 & 7 & 3\end{array}\right),\left(\begin{array}{lll}1 & 7 & 2\end{array}\right),\left(\begin{array}{lll}1 & 7 & 1\end{array}\right),\left(\begin{array}{lll}2 & 7 & 1\end{array}\right),\left(\begin{array}{lll}3 & 7 & 1\end{array}\right)$, (4 $\left.\left.7 \begin{array}{ll}1\end{array}\right),\left(\begin{array}{llll}5 & 7 & 1\end{array}\right),\left(\begin{array}{llll}6 & 7 & 1\end{array}\right),\left(\begin{array}{lll}8 & 7 & 1\end{array}\right),\left(\begin{array}{lll}9 & 7 & 1\end{array}\right)\right\}$. 


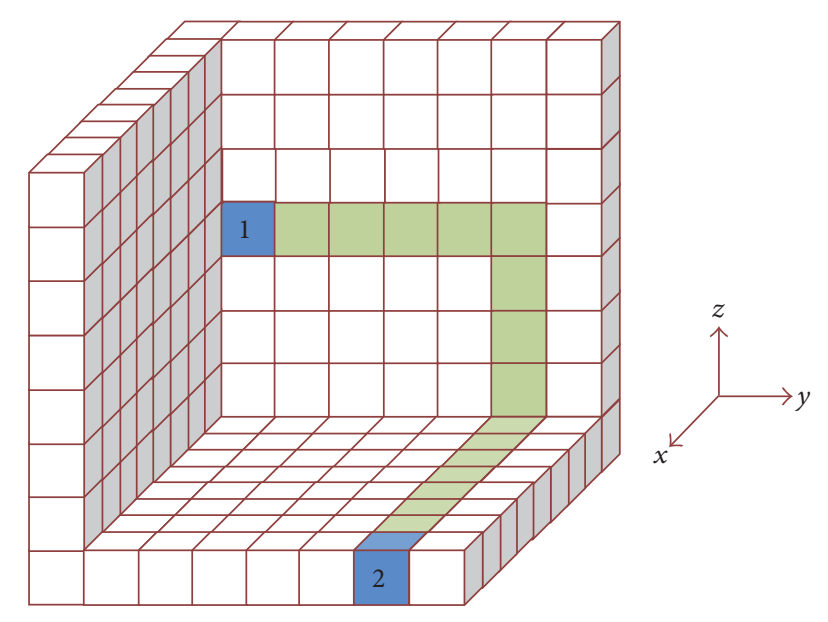

Object point

Route path

FIgURE 3: Definition of pipe path.

2.2.3. Representation of Pipeline. During the ship pipe route planning, different diameters of pipelines may be required to connect different equipment; for example, diameter values of the pipes connecting fuel oil storage tanks are larger than those of pipes connecting marine main engines. In consideration of the differences between pipeline diameters, the concept of pipe grading is introduced. If all pipes in a route path are sorted by the diameter values, the top one, namely, the pipe with largest diameter value, is defined as pipe grade 1 , whose terminals are defined as points-grade 1 , and the remaining pipes can be graded successively according to the ranked list.

Since the workspace is approximately described by cube grid method, the accuracy depends on the size of cube grid. In this paper, the minimum round pipe diameter of all pipelines in the design space is chosen as the cube side length. For a pipeline, including different diameter of pipes, to simplify the path routing algorithm, the border cells of obstacles including the generated pipe lines are extended outward by an appropriate cell number. By this method, the encoding of subpipe grade with larger diameter remains the same with that of the subpipe grade with smaller diameter. The extended cell number is determined by largest round pipe diameter of current pipeline, as shown in

$$
n= \begin{cases}\operatorname{int}\left(\frac{D_{\max }}{2 L}\right)+1, & \frac{D_{\max }}{2 L}-\operatorname{int}\left(\frac{D_{\max }}{2 L}\right)>0.5 \\ \operatorname{int}\left(\frac{D_{\max }}{2 L}\right), & \frac{D_{\max }}{2 L}-\operatorname{int}\left(\frac{D_{\max }}{2 L}\right) \leq 0.5\end{cases}
$$

where $n$ represents the cell number that needs to be extended by obstacles, $D_{\max }$ represents the largest diameter of current pipeline, and $L$ denotes side length of cells. Once a pipeline is routed, the generated pipeline will be set as obstacles. Then, the mathematical model of workspace will be updated according to the parameters of next pipeline that need to be routed.

2.3. Problem Formulation of Pipe Routing. Ship pipe route planning is a typical multiobjective optimization problem; that is, it aims to achieve optimization for the best compromise pipeline under several given constraints based on the discrete mathematical model. The considered constraints and evaluation criteria are as follows.

(i) Avoiding obstacles;

(ii) Shortest length of route path;

(iii) Minimum bends;

(iv) Maximum overlapping length of subpipe route paths.

Then the objective functions $\operatorname{Obj}(f)$ for pipeline design are determined as follows:

$$
\begin{array}{ll}
\min & \operatorname{Obj}\left(f_{1}\right)=L_{P}, \\
\min & \operatorname{Obj}\left(f_{2}\right)=B_{P}, \\
\max & \operatorname{Obj}\left(f_{3}\right)=O_{P},
\end{array}
$$

where $L_{P}$ represents the length of the pipeline, namely, the number of grid cells passed through by the axis of pipeline from starting point to end point, $B_{P}$ represents the number of bends, and $O_{P}$ denotes the overlapped length between current pipe grade and other pipe grades.

\section{Proposed Pipe Routing Method}

3.1. Overview of the Proposed Method. According to the connection relationships of equipment as depicted in schematic diagram of piping system, the terminal set of each pipeline is obtained. In consideration of the effect of pipeline diameter on layout result and usage safety, the pipe grading is conducted, and then diameter value of subpipe grade 1 is taken as the typical diameter value of each pipeline with certain subpipes. And then the rules for piping layout sequence are decided as follows.

(1) The pipeline with larger diameter is arranged in priority.

(2) For subpipes of a pipeline, the one with higher grade is arranged in priority.

(3) Branch point is generated on the subpipe with adjacent higher grade.

An example of a pipeline with three subpipes is shown in Figure 4. Regardless of the difference of subpipe diameters, the routing result is shown in Figure 4(a). When the pulsating pressure exists in pipeline, the excitation force will be generated at the branch point due to the large difference between diameter values of subpipe grade 1 and subpipe grade 3, which will result in vibration influencing working period and usage security of the pipeline. By considering the diameter difference of subpipes, the routing result is improved based on the order of arrangement, which is shown in Figure 4(b). 

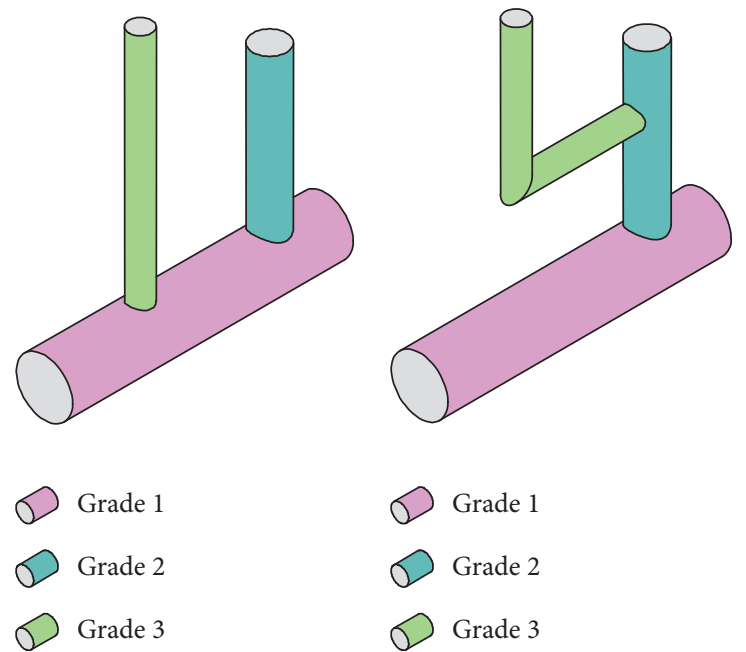

(a)

(b)

FIGURE 4: Selection of branch point location.

According to the mentioned rules for pipe routing design, a ship pipe routing methodology is proposed by combining MA, NSGA-II, and CCNSGA-II. The flow chart of the proposed method is shown in Figure 5, where MANSGA-II denotes the combination of MA and NSGA-II, and MA-CCNSGA-II represents the combination of MA and CCNSGA-II.

For case with two terminals, MA-NSGA-II is used for pipe routing design as shown in Figure 6. The search strategy of tendencies in direction is mainly adopted by researchers to generate initial population, which might lead to the generation of repeated nodes in a chromosome. In this paper, maze algorithm is used to generate the initial individuals to avoid generating repeated nodes. Since fewer individuals are required to compose the initial population, the search efficiency of maze algorithm becomes acceptable. The Pareto optimal set is obtained after optimizing the initial population by using NSGA-II algorithm, and fuzzy set theory is used to select the best compromise solution of current subpipe, which will be referred during the optimization procedure of other subpipes.

For cases with three or more terminals, MA-CCNSGAII is used for pipe routing design as shown in Figure 7. Decomposition method, namely, the optimization problem, is divided into several subproblems to be optimized, respectively, and is adopted to deal with multibranch pipeline routing problem. The key connecting point is taken as the starting point, and other points are taken as end points, respectively. The initial subpopulations are generated in turn by using the same method in the cases with two terminals. And cooperative coevolutionary strategy is applied in optimization algorithm, in which the subproblems are optimized cooperatively. Therefore, the optimal individual of each subpopulation is selected to be shared during the solution procedures, and an individual of a certain subpopulation is evaluated by calculating the fitness function of the solution constructed by the individual and optimal individuals of other subpopulations. The evolution and cooperation of each population are performed continually until the evolution is completed, and the final optimal individuals are combined together as an optimal solution of the multibranch pipes.

The key connecting point is defined as follows. For pointsgrade 1, the sum of Euclidean distances between a certain point of points-grade 1 and others is, respectively, calculated by (5), and the point with minimum sum is selected as key connecting point of pipe grade 1 ; for points-grade $i(i>1)$, the key connecting point is generated in points-grade $i-1$. The sum of Euclidean distances between a certain point of points-grade $i-1$ and the connecting points of points-grade $i$ is calculated by (5), respectively. Then, the point of pointsgrade $i-1$ with minimum sum is taken as key connecting point of pipe grade $i$.

$$
L_{P_{i}}=\sum_{j=1}^{n} L_{P_{i} P_{j}}, \quad i=1,2, \ldots, n ; j=1,2, \ldots, n,
$$

where $L_{P_{i}}$ represents the sum of Euclidean distances when the connecting point $P_{i}$ is taken as the starting point; $L_{P_{i} P_{j}}$ denotes the Euclidean distance between connecting points $P_{i}$; and $P_{j} ; n$ represents the number of connecting points. An example for the key connecting point selection of pipeline with three subpipe grades is shown in Figure 8. As shown in Figure 8, according to the concept of pipe grading, the connecting points $P_{1}$ and $P_{2}$ are defined as points-grade 1 , the connecting points $P_{3}$ and $P_{4}$ are defined as points-grade 2 , and $P_{5}$ is defined as points-grade 3 . Then, according to the definition of key connecting point, $P_{1}$ is selected as key connecting point of subpipe grade 1 and subpipe grade 2 , and $P_{4}$ is selected as key connecting point of subpipe grade 3 .

3.2. Adaptive Region. Due to the huge piping layout space, global search for optimal pipeline will result in operation with large storage and low efficiency. So the adaptive 


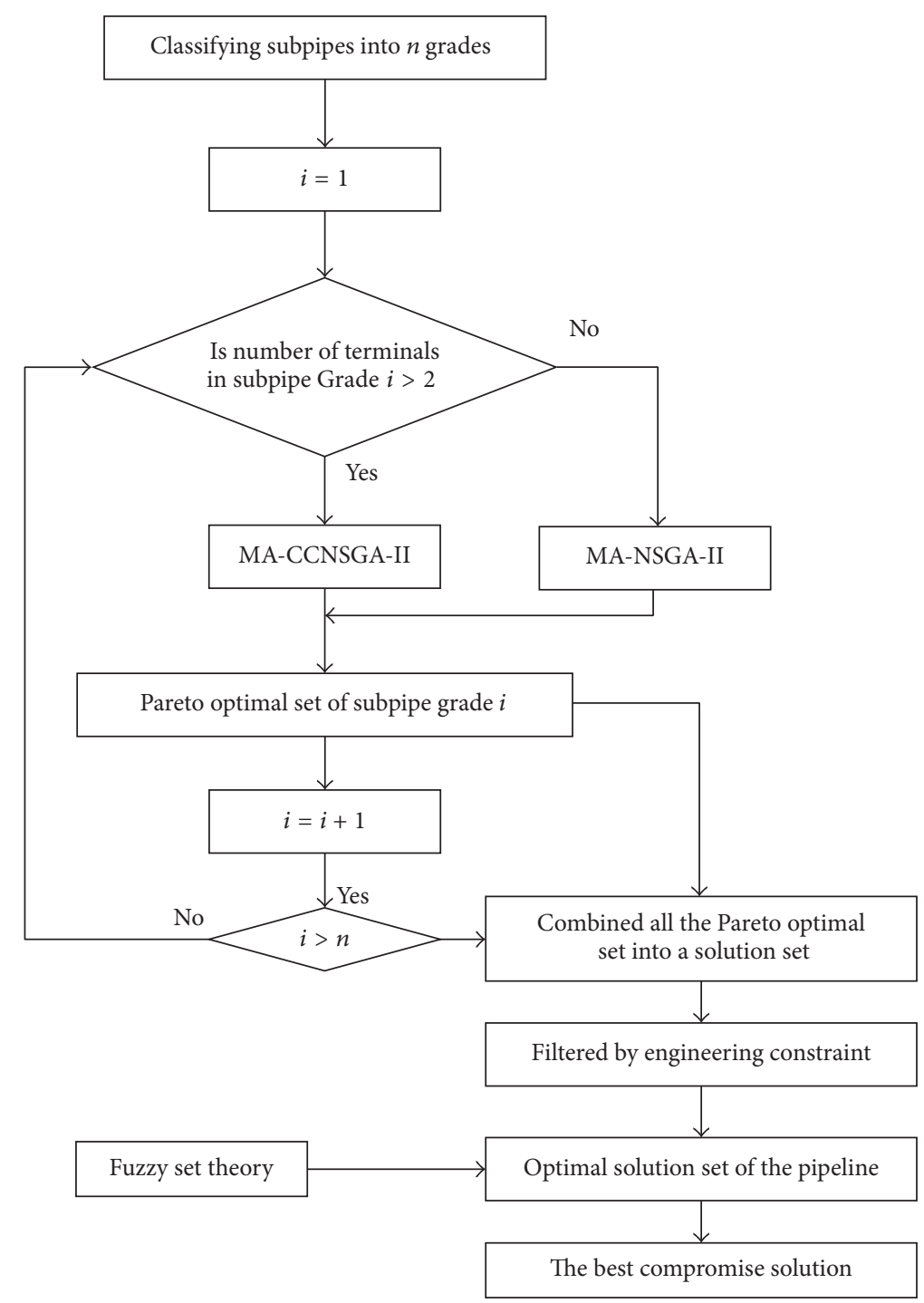

FIGURE 5: The flow chart of ship pipe routing algorithm based on pipe grading.

region strategy [33] is extended for application in threedimensional space to limit the searching scopes, by which a more compact layout is also guaranteed. Especially, as shown in Figure 9, given $n$ pipe terminals with coordinates $P_{1}\left(x_{1}, y_{1}, z_{1}\right), P_{2}\left(x_{2}, y_{2}, z_{2}\right), \ldots, P_{n}\left(x_{n}, y_{n}, z_{n}\right)$, the local search space can be determined by two points $A\left(x_{A}, y_{A}, z_{A}\right)$ and $B\left(x_{B}, y_{B}, z_{B}\right)$ formulated by (6), where $k$ is a constant, and $k \geq 0 ; \Delta x, \Delta y$ and $\Delta z$ are, respectively, the expanded sizes in $x$-, $y$-, and $z$-axes, which are determined by trial and error.

$$
\left[\begin{array}{l}
x_{A} \\
y_{A} \\
z_{A} \\
x_{B} \\
y_{B} \\
z_{B}
\end{array}\right]=\left[\begin{array}{l}
\min \left\{x_{1}, x_{2}, \ldots, x_{n}\right\} \\
\min \left\{y_{1}, y_{2}, \ldots, y_{n}\right\} \\
\min \left\{z_{1}, z_{2}, \ldots, z_{n}\right\} \\
\max \left\{x_{1}, x_{2}, \ldots, x_{n}\right\} \\
\max \left\{y_{1}, y_{2}, \ldots, y_{n}\right\} \\
\max \left\{z_{1}, z_{2}, \ldots, z_{n}\right\}
\end{array}\right]+k \cdot\left[\begin{array}{l}
-\Delta x \\
-\Delta y \\
-\Delta z \\
+\Delta x \\
+\Delta y \\
+\Delta z
\end{array}\right] .
$$

\subsection{Improved Maze Algorithm}

3.3.1. Extended Search. Extended search is the expansion process starting from one grid to the adjacent grid, and a grid is specified by its tag value marked in the process. The rules of marking tag value are as follows.

(1) Initial grid is marked as "1."

(2) Only six neighbors of the current grid could be marked, whose tag value is that of current grid plus 1.

(3) If the adjacent grid has been marked, the smaller tag value is selected.

An expansion process diagram is illustrated in Figure 10, in which the blue grid cell $\left(\begin{array}{lll}1 & 1 & 10\end{array}\right)$ is starting point and the red grid cell $\left(\begin{array}{lll}9 & 8 & 1\end{array}\right)$ is goal point. In the space built by the starting point and the goal point, gray cells marked as "\#" represent obstacles in the layout space, the tag value of the 


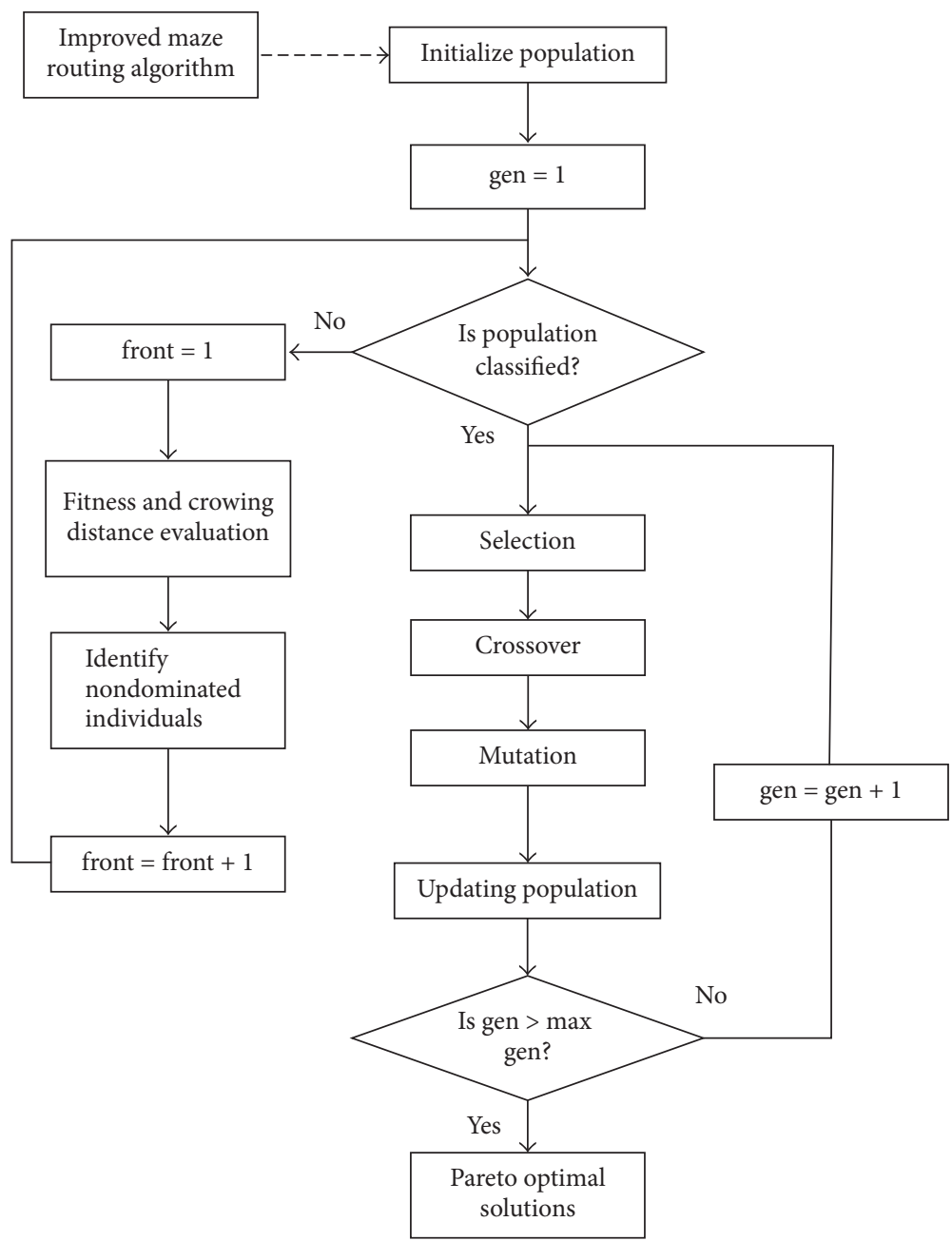

Figure 6: The flow chart of MA-NSGA-II.

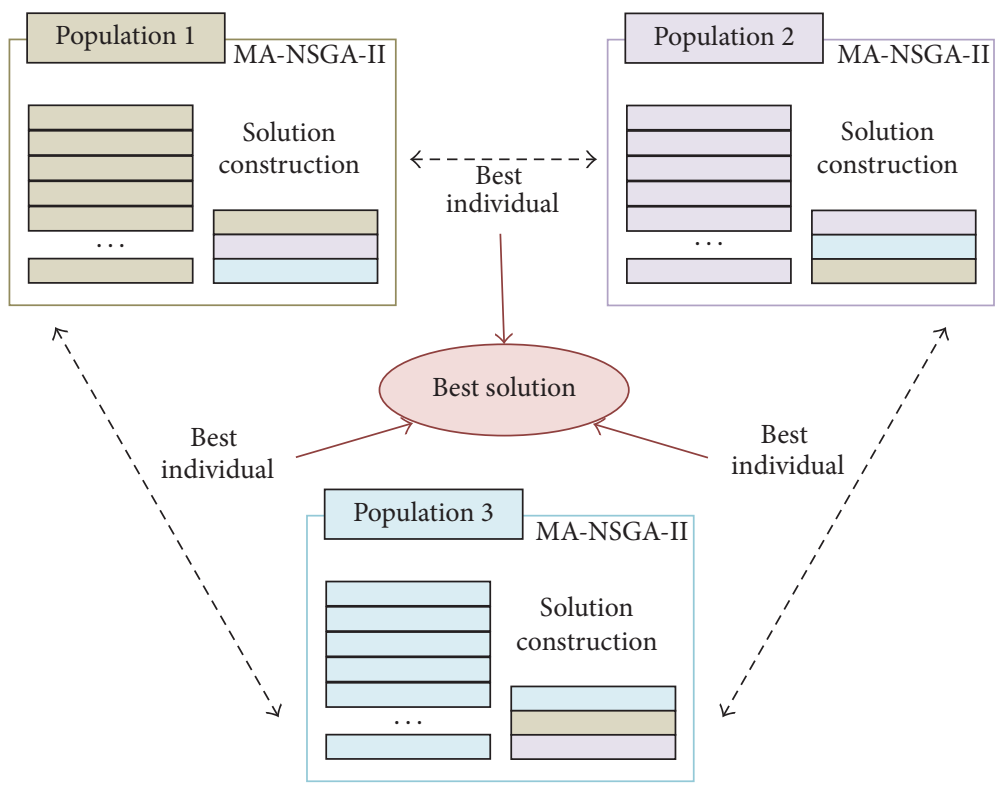

Figure 7: The schematic diagram of MA-CCNSGA-II. 


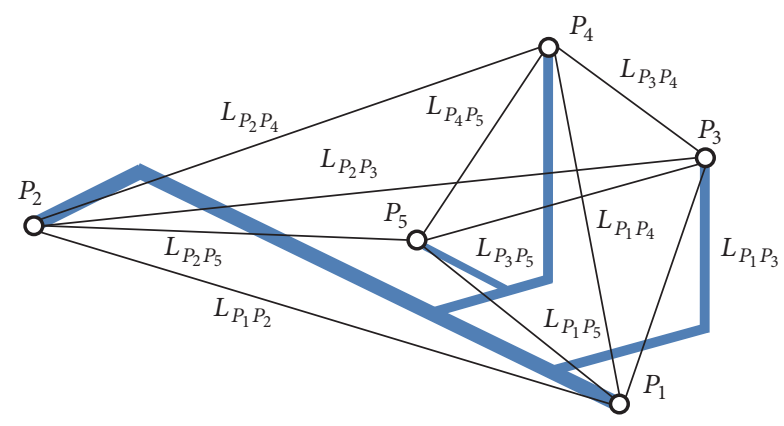

Figure 8: An example for the key connecting point selection.

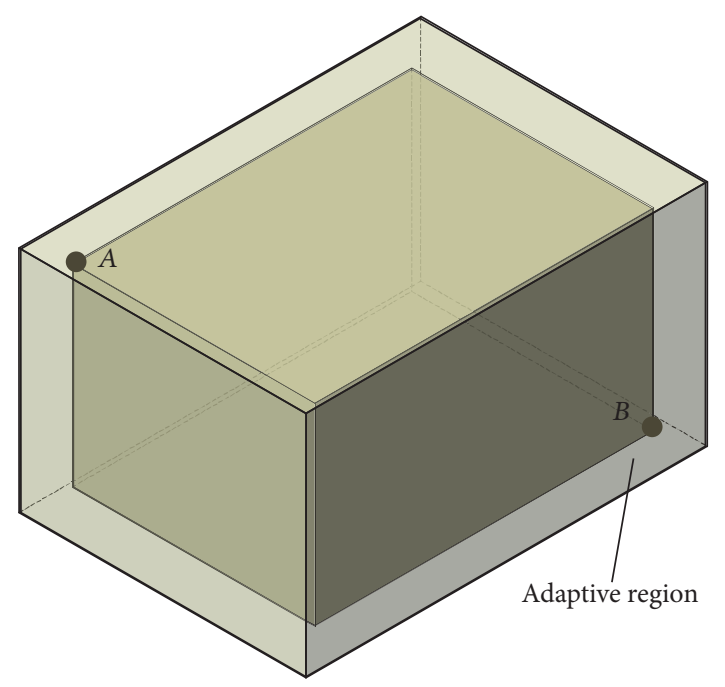

FIgURE 9: Adaptive region in 3D space.

starting point is 1 , and the tag values of six adjacent grids are 2 . The expansion process will be continued until the goal point whose tag value is 22 has been reached.

3.3.2. Retracing. Retracing process is antisearch from goal point to starting point. Figure 11 shows a retracing process from the red grid cell $\left(\begin{array}{lll}9 & 8 & 1\end{array}\right)$ to the blue grid cell $\left(\begin{array}{lll}1 & 1 & 10\end{array}\right)$. According to the coordinates of blue and red grid cells, the direction vectors of antisearch are determined: $\left(\begin{array}{lll}-1 & 0 & 0\end{array}\right)$, $\left(\begin{array}{lll}0 & -1 & 0\end{array}\right)$, and $\left(\begin{array}{lll}0 & 0 & 1\end{array}\right)$. Starting from the red grid cell, a random searching direction vector is determined as the retracing direction, and then the found grid cell with smaller tag value will be then chosen as the current starting point; the retracing process is completed when the grid cell marked " 1 " is found. For the cells marked as obstacles, search direction will be changed randomly until a feasible grid cell is found. Finally, the initial population with a specific number of individuals is generated by repeated retracing process.

3.3.3. Introduction of Auxiliary Point. When the workspace is marked by using depth-first search strategy, the adaptive region cannot be reached entirely by the retracing process, which limits the diversity of population and affects the optimization efficiency, and then the auxiliary point is introduced to solve this problem. An example for two-dimensional maze search is shown in Figure 12. The green and blue grids are the starting and goal points, respectively, and the tag values of grid cells in search space can be marked by the extended search process. Since retracing process searches towards grids with decreasing tag values, it is obvious that pipe paths mainly appear on the upper right area though feasible pipe paths can be found on the lower left area. Although it has little impact on the single pipeline, current optimal path may be not the optimal pipe path when there exists other pipelines, such as the pipe of same grade existed. Therefore, the auxiliary point which is chosen randomly inside the adaptive region is added into the lower left area, as the red grids shown in Figure 12. By taking the green grid as the starting point and the auxiliary grid as the goal point, pipe path 1 is generated by using maze algorithm. Then pipe path 2 is generated by taking the auxiliary grid as the start point and the blue grid as the goal point, and the pipe path is finally generated by connecting path 1 and path 2. Apparently, total length of current grade pipe is reduced by introducing the auxiliary point.

As to the introduction of auxiliary point, the distribution scope of pipe paths is expanded and the diversity of initial population is increased, which provides the basis for better optimization.

3.4. Fixed-Length Encoding. To overcome the drawbacks of variable-length encoding technique, such as the complexity in dealing with chromosomes and generating chromosomes with repeated nodes in genetic operation, a fixed-length encoding method is proposed based on the improved maze algorithm and extended adaptive region strategy, which improves the performance in genetic algorithm procedure.

Two-dimensional diagram shown in Figure 13 is taken as an example to describe the fixed-length encoding method. Taking point 1 as the starting grid and point 2 as the goal grid, then the tag value of point 2 represents the maximum length of the feasible pipe path. Based on the added auxiliary point, searching space is extended into the whole adaptive region. While the furthest point compared to points 1 and 2 is chosen as the auxiliary point, as the red point shown in Figure 13, the maximum length of pipe path is the maximum tag value plus $2(\Delta x+\Delta y)$ and larger than the original length regardless of the obstacles in the extended space. While considering the obstacles, the maximum length of feasible pipe path is the sum of the tag values of two separated subpaths. For subpath 1 , point 1 is taken as starting point and the added auxiliary point is taken as goal point; then the tag value of the auxiliary point denotes the length of subpath 1 . For subpath 2, the auxiliary point is taken as starting point and point 2 is taken as goal point; then the tag value of point 2 denotes the length of subpath 2 .

Due to the large layout space in a ship and separate distribution of obstacles, we assume that the extended space is a workspace without obstacles. Therefore, the length of chromosome in $3 \mathrm{D}$ space is determined as the maximum tag value plus $2(\Delta x+\Delta y+\Delta z)$, where $\Delta x, \Delta y$, and $\Delta z$ are, respectively, the max expanded sizes in $x$-, $y$ - and $z$-axes. 


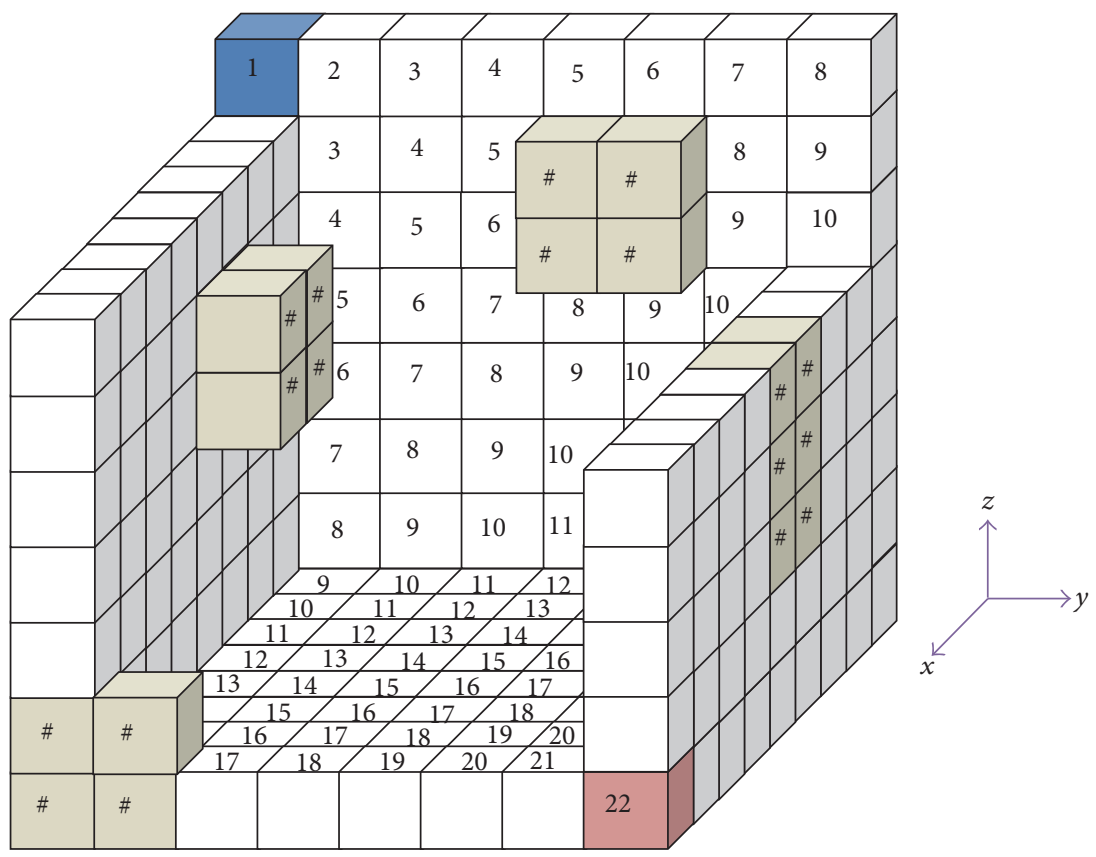

Starting point
\# Cells on obstacle

FIGURE 10: An example for the extended search process of maze algorithm.

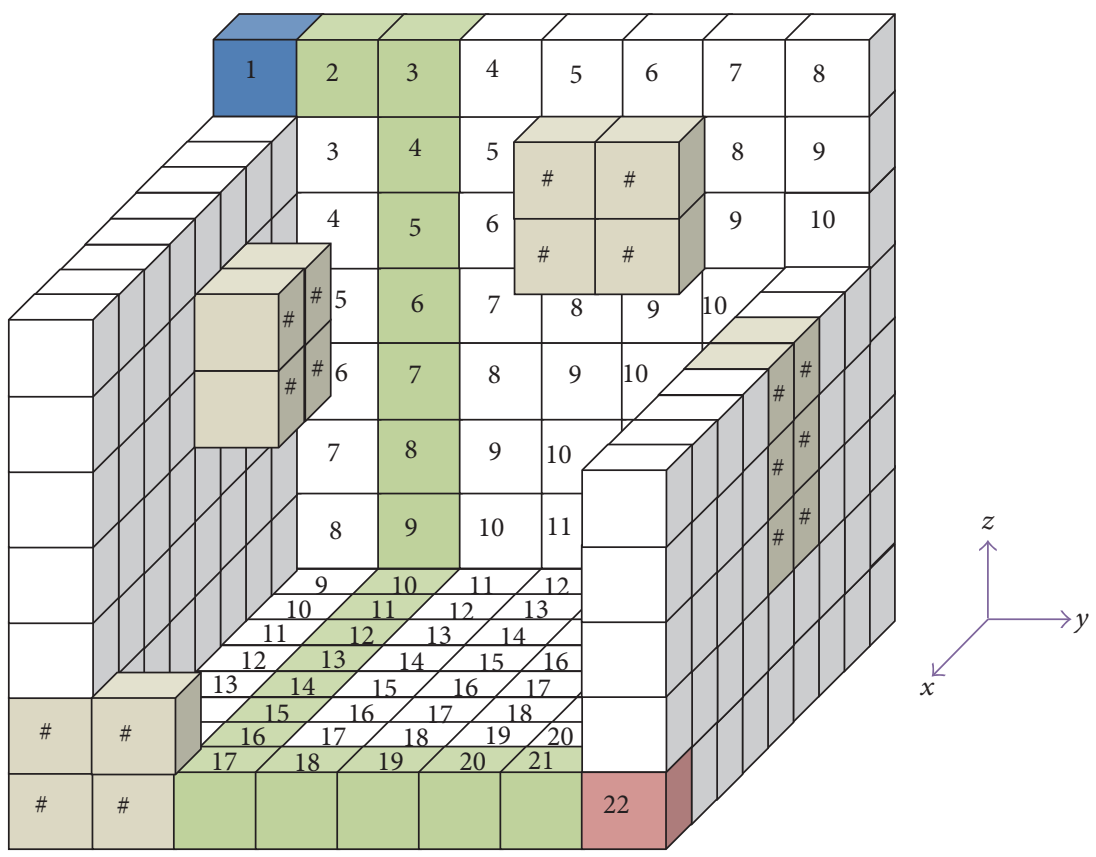

Starting point \# Cells on obstacle

Goal point $\square$ Route path

FIGURE 11: An example for retracing process of maze algorithm. 


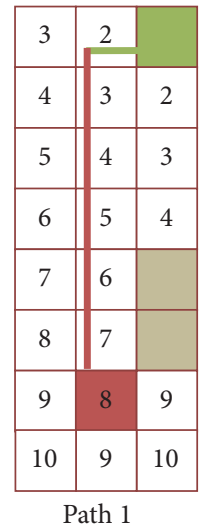

\begin{tabular}{|c|c|c|c|c|c|c|c|c|c|}
\hline 4 & 3 & 2 & 3 & 4 & 5 & 6 & 7 & 8 & 9 \\
\hline 3 & 2 & & 2 & 3 & 4 & 5 & 6 & 7 & 8 \\
\hline 4 & 3 & 2 & 3 & 4 & 5 & 6 & 7 & 8 & 9 \\
\hline 5 & 4 & 3 & 4 & & & 7 & 8 & 9 & 10 \\
\hline 6 & 5 & 4 & 5 & & & 8 & 9 & 10 & 11 \\
\hline 7 & 6 & & & 11 & 10 & 9 & 10 & 11 & 12 \\
\hline 8 & 7 & & & 12 & 11 & 10 & 11 & 12 & \\
\hline 9 & 8 & 9 & 10 & 11 & 12 & $\times 11$ & & & \\
\hline 10 & 9 & 10 & 11 & 12 & 13 & 12 & & & \\
\hline 11 & 10 & 11 & 12 & & & & & & \\
\hline
\end{tabular}
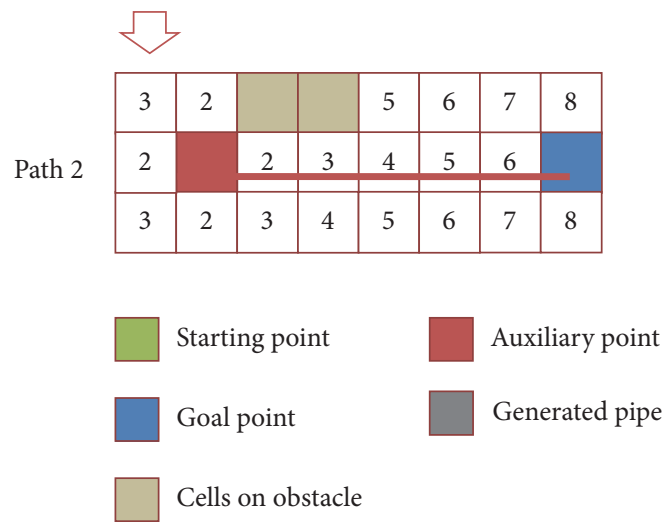

FIGURE 12: Introduction of auxiliary point.

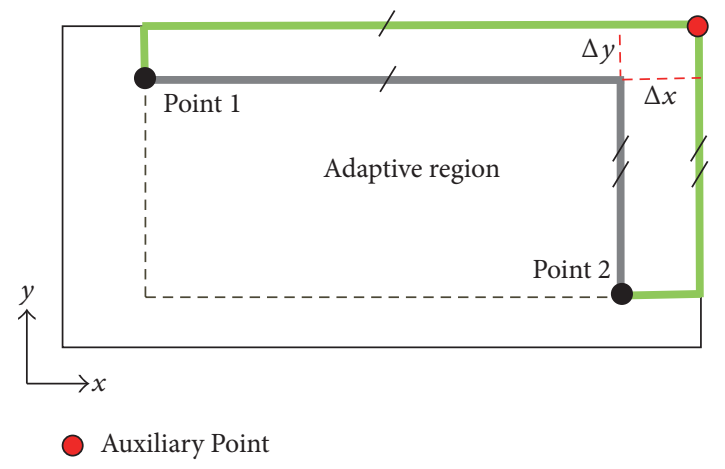

FIGURE 13: Fixed-length encoding method.

3.5. Fitness Function. Fitness value is the evaluation criterion for superiority of a chromosome and the basis of nondominated sorting and selection operation in nondominated sorting genetic algorithm. The objective function formulated in Section 2.3 is directly taken as the fitness function of optimization. Eqs. (2) and (3) are taken as fitness functions for optimization problem of pipeline with two terminals, while (2), (3), and (4) are taken as the fitness functions for optimization problem of pipeline with multiterminals.

An example of pipeline with three subpipe grades is shown in Figure 14. For subpipe grade 1, the length is 11 and the number of bends is 0 ; the overlapping length with subpipe grade 2 and subpipe grade 3 is 8 and 4, respectively. As for subpipe grade 2, two subpaths are included; the total length is 24 and the number of bends is 4; the overlapping length with subpipe grade 1 and subpipe grade 3 is 8 and 15, respectively. For subpipe grade 3 , the length is 16 and the number of bends is 4 ; the overlapping length with subpipe grade 1 and subpipe grade 2 is 4 and 15, respectively. Then, for the pipeline, the total length is 28 and the number of bends is 5 (3 T-junctions included).

3.6. Genetic Operator. Only the pipe path chromosome, which is composed of a series of nodes with continuous coordinates, can be regarded as a valid one. By using traditional genetic operator directly in crossover and mutation operation, the connectivity of nodes in a chromosome cannot be guaranteed after genetic operation, which may lead to the generation of invalid individuals. Based on the fixed-length encoding method mentioned in Section 3.4, the crossover and mutation operation method with fixed-length chromosome is introduced by improving the traditional genetic operator.

3.6.1. Crossover. The crossover point is selected randomly in the traditional single-point crossover operation, and then two offspring individuals are generated by exchanging the 
Subpipe grade 1

\begin{tabular}{|l|l|l|l|l|l|l|l|l|l|l|}
\hline 133 & 233 & 333 & 433 & 533 & 633 & 733 & 833 & 933 & 1033 & 1133 \\
\hline
\end{tabular}

\begin{tabular}{|c|c|c|c|c|c|c|c|c|c|c|c|c|c|c|c|}
\hline \multicolumn{16}{|c|}{ Subpath 1} \\
\hline 133 & 233 & 333 & 433 & 533 & 543 & 553 & 563 & 573 & 583 & 584 & 585 & 586 & 000 & 000 & 000 \\
\hline \multicolumn{16}{|c|}{ Subpath 2} \\
\hline 133 & 233 & 333 & 433 & 533 & 633 & 733 & 833 & 843 & 853 & 863 & 873 & 883 & 884 & 885 & 886 \\
\hline \multicolumn{16}{|c|}{ Subpipe grade 3} \\
\hline 586 & 585 & 584 & 583 & 573 & 563 & 553 & 543 & 533 & 633 & 733 & 833 & 843 & 853 & 863 & 963 \\
\hline
\end{tabular}

FIGURE 14: An example for fitness evaluation.

Parent 1

\begin{tabular}{|c|c|c|c|c|c|c|c|c|c|c|c|c|}
\hline 154 & 164 & 174 & 184 & 194 & 294 & 394 & 494 & 594 & 595 & 596 & 586 & 576 \\
\hline \multicolumn{13}{|c|}{ Parent 2} \\
\hline 154 & 254 & 354 & 454 & 554 & 564 & 574 & 575 & 576 & 000 & 000 & 000 & 000 \\
\hline \multicolumn{13}{|c|}{ Mid-Path } \\
\hline & & & & 174 & 274 & 374 & 474 & 574 & & & & \\
\hline \multicolumn{13}{|c|}{ Child 1} \\
\hline 154 & 164 & 174 & 274 & 374 & 474 & 574 & 575 & 576 & 000 & 000 & 000 & 000 \\
\hline \multicolumn{13}{|c|}{ Child 2} \\
\hline 154 & 254 & 354 & 454 & 554 & 564 & 574 & 474 & 374 & 274 & 174 & $\ldots$ & 576 \\
\hline
\end{tabular}

FIGURE 15: Crossover based on fixed-length encoding method.

right parts of two parent chromosomes. A crossover strategy with fixed-length chromosomes is proposed by improving single-point crossover operator. For two chromosomes, two crossover nodes, $\left(x_{i}, y_{i}, z_{i}\right)$ and $\left(x_{j}, y_{j}, z_{j}\right)$, which are not the starting and goal nodes in two chromosomes, are selected, and an assistant path is generated by taking $\left(x_{i}, y_{i}, z_{i}\right)$ and $\left(x_{j}, y_{j}, z_{j}\right)$ as the starting and goal points. Then the offspring chromosomes are generated by adding the assistant path into the parent chromosomes whose right parts are exchanged with each other. The offspring chromosome will be deleted if its length exceeds the limited length of fixed-length chromosome.

The generation method for assistant paths is different from that for initial population. To improve the operation efficiency of the algorithm and avoid generating repeated nodes, the following method is applied: the extended search process of maze algorithm is still used to encode the workspace composed by start and goal points. Then the prior search direction is determined by the position relationship between two points, and an initial search direction is chosen randomly to search towards the grid cells with decreasing values. The search direction will not be changed unless the grid cell on obstacle is searched, and process will be continued until the terminal point is reached.
As shown in Figure 15, the encodings of parent chromosomes Parent 1 and Parent 2 are illustrated. Nodes number 3 and 7 are, respectively, the crossover points for Parent 1 and Parent 2, and the coordinates of crossover points are $(1,7,4)$ and $(5,7,4)$. As depicted above, an assistant path (as Mid-path in Figure 15) is generated, and the parent chromosomes are rebuilt to generate the offspring chromosomes: Child 1 and Child 2. In this case, the length of chromosome Child 1 is less than the given length, and the empty nodes are supplemented by " 0 ." On the contrary, the length of chromosome Child 2, which exceeds the limited length, will be deleted directly.

3.6.2. Mutation. Similar to the crossover operation, the connectivity of pipe path cannot be guaranteed by using traditional mutation operation method directly. A mutation strategy of fixed-length chromosome is given in this part. For the parent chromosome, two mutation points are randomly selected as the starting and end points, respectively, and an assistant path is generated by the same way as that in crossover operation. Then the original nodes between two mutation points are replaced by the assistant path, which brings the new offspring chromosome. Similarly, if the length of offspring chromosome exceeds the limited length, it will 
Parent

\begin{tabular}{|l|l|l|l|l|l|l|l|l|l|l|l|l|l|}
\hline 154 & 164 & 174 & 184 & 194 & 294 & 394 & 494 & 594 & 595 & 596 & 586 & 576 \\
\hline
\end{tabular}
\begin{tabular}{|l|l|l|l|l|l|l|l|l|l|l|l|l|}
\hline 174 & 184 & 284 & 384 & 484 & 584 & 585 & 586 \\
\hline 154 & 164 & 174 & 184 & 284 & 384 & 484 & 584 & 585 & 586 & 576 & 000 & 000 \\
\hline
\end{tabular}

FIGURE 16: Mutation based on fixed-length encoding method.

TABLE 1: Information of piping route path and connecting points.

\begin{tabular}{lcc}
\hline Route path name & Coordinates of connecting points & Pipe specification $(\mathrm{mm})$ \\
\hline Pipe grade 1 & $(2,2,2),(10,44,46)$ & 20 \\
Pipe grade 2 & $(39,12,48)$ & 15 \\
Pipe grade 3 & $(45,46,15)$ & 10 \\
\hline
\end{tabular}

be removed, or else the empty nodes will be supplemented by “0."

An example for mutation operation is shown in Figure 16, in which encoding of the parent chromosome Parent is illustrated. Nodes number 3 and 12 are selected as two mutation points, and then the assistant path Mid-path is generated based on their coordinates $(1,7,4)$ and $(5,8$, $6)$. Finally, the offspring chromosome Child is produced by replacing the nodes between two mutation points with Midpath, and the empty nodes are supplemented by " 0. ."

3.7. Fuzzy Set Theory. For Pareto optimal chromosome sets with $N_{\text {obj }}$ objectives and $M$ individuals, a membership function $\mu_{i}$ denotes the $i$ th objective function of an individual in Pareto optimal solutions, which is defined in [39]:

$$
\mu_{i}= \begin{cases}1, & F_{i} \leq F_{i}^{\min }, \\ \frac{F_{i}^{\max }-F_{i}}{F_{i}^{\max }-F_{i}^{\min }}, & F_{i}^{\min }<F_{i}<F_{i}^{\max }, \\ 0, & F_{i} \geq F_{i}^{\max },\end{cases}
$$

where $F_{i}^{\max }$ and $F_{i}^{\mathrm{min}}$, respectively, denote the maximum and minimum values of the $i$ th fitness function. For each nondominated solution $k$, the normalized membership function $\mu^{k}$ is expressed as follows:

$$
\mu^{k}=\frac{\sum_{i=1}^{N_{\mathrm{obj}}} \mu_{i}^{k}}{\sum_{j=1}^{M} \sum_{i=1}^{N_{\mathrm{obj}}} \mu_{i}^{j}} .
$$

In (8), larger $\mu^{k}$ indicates better compromise individual. Therefore, a priority list of nondominated solutions are obtained by descending sort of $\mu^{k}$, and the optimal individual is easily determined.

\section{Case Studies}

4.1. Case Study 1: A Design Example of Branch Pipeline. To verify the feasibility and efficiency of the proposed algorithm, the proposed approach is utilized to execute a branch pipe design example of our previous work [40].

4.1.1. Parameter Settings of Model Space. The pipe routing space is set as $500 \mathrm{~mm} \times 500 \mathrm{~mm} \times 500 \mathrm{~mm}$ and is divided by using the cube whose side length is $10 \mathrm{~mm}$. the working space is then divided into $50 \times 50 \times 50$ uniform $3 \mathrm{D}$ cubic grid cells. Obstacles in the workspace are represented by the cuboids whose diagonal coordinates are determined as follows: $\{(5,1$, 5), (15, 51, 15); (1, 27, 29), (30, 42, 44); (1, 1, 35), (21, 20, 50); $(30,5,1),(45,40,20) ;(32,1,25),(47,20,40) ;(32,1,40),(47$, $8,50)\}$. The coordinates of the connection points involved in the branch pipe and the assumed pipe specification are listed in Table 1.

4.1.2. Optimization Procedure. In this example, each pipe grade includes two connecting points, and MA-NSGA-II is adopted as the pipe routing algorithm. Based on the concept of key connecting point given in Section 3.1, for subpipe grades 1 and 2 , the point $(2,2,2)$ is selected as the key point. For subpipe grade 3 , the point $(39,12,48)$ is selected as the key point. As motioned in Section 2.2.3, for subpipe grades 1 and 2, the border cells of obstacles are extended outward by one grid accordingly, to satisfy computing requirements of larger diameter grades. Accordingly, three initial populations are generated by modified Maze algorithm. The optimization procedure is conducted by the sequence of pipe grades 1 , 2 , and 3. The length and bending numbers of the pipeline are taken as the optimization objects for each subpipe grade, and the parameters of MA-NSGA-II are listed in Table 2.

The evolution graphs of average and minimum values of the two objects versus generation are, respectively, illustrated in Figures 17-19. As depicted in the figures, both the average and the minimum values of length and bending numbers of subpipe grades are trending to decrease with increasing evolution generation by using MA-NSGA-II. It also indicates that individuals converge to the some appropriate route after 


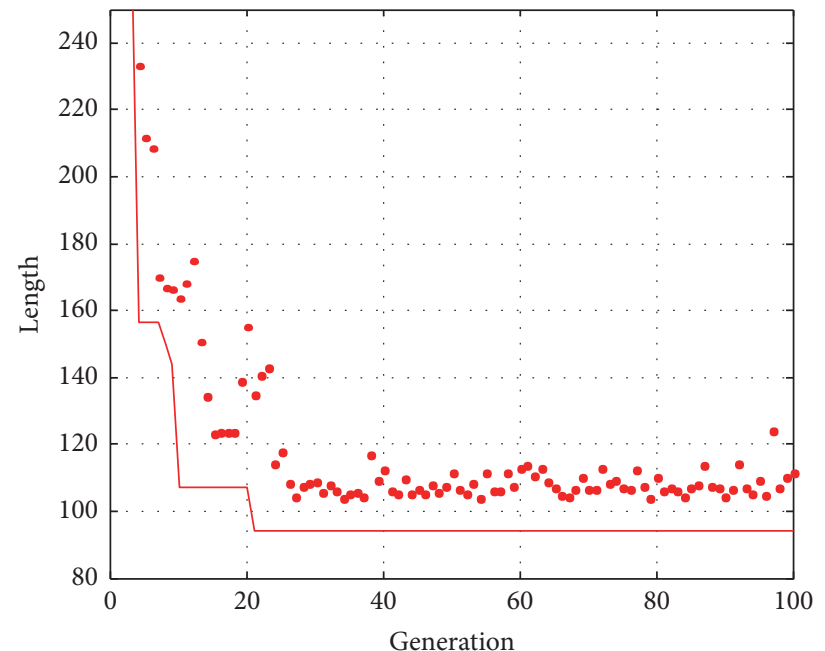

Minimum
Average

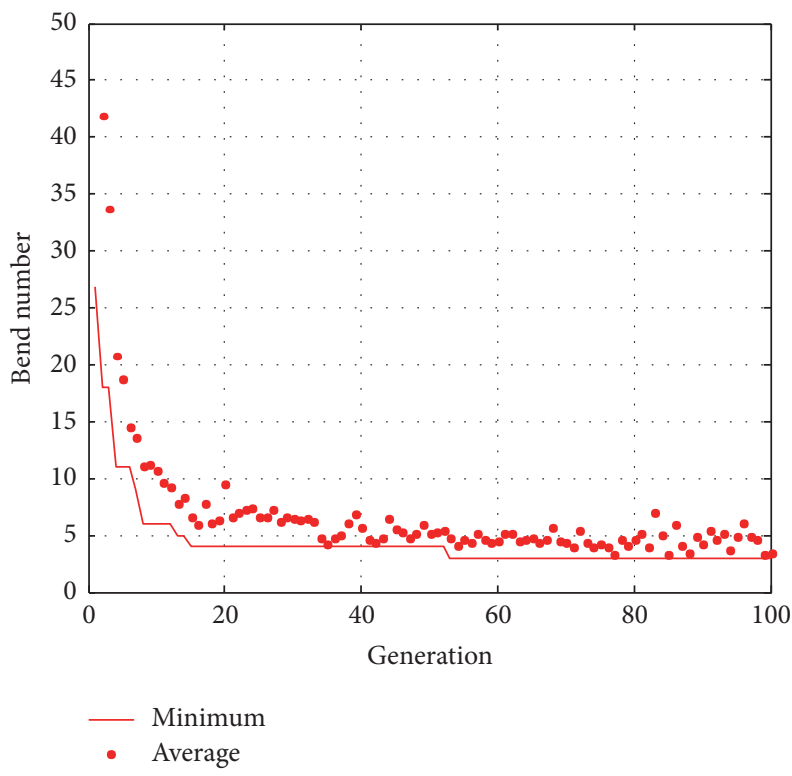

(b)

(a)

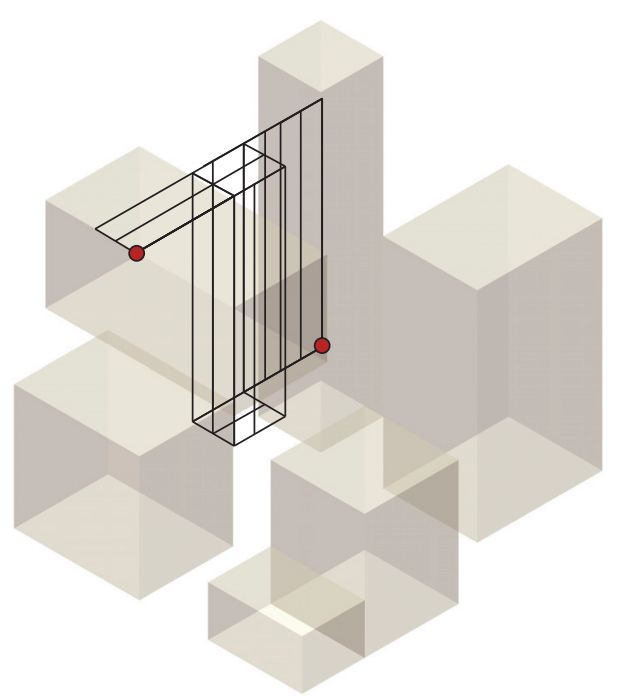

(c)

Figure 17: Evolutionary graphs of pipe grade 1. (a) Evolution process of length; (b) evolution process of bend numbers; (c) Pareto optimal set.

TABLE 2: Parameters of MA-NSGA-II.

\begin{tabular}{lc}
\hline Parameter & Value \\
\hline Population size & 50 \\
Number of generation & 100 \\
Crossover probability & 0.8 \\
Mutation probability & 0.05 \\
\hline
\end{tabular}

around 60th generation. Pareto optimal set of three subpipe grades are depicted in Figures 17(c), 18(c) and 19(c).

4.1.3. Optimal Solution Set of the Branch Pipeline. As shown in Figure 5, optimal solution set of the branch pipeline is constructed as follows: Firstly, the Pareto solution sets of each subpipe grade are combined into a solution set for the branch pipeline. Feasible solutions for the branch pipeline is generate by permutation and combination of all individuals of different Pareto solution sets. Then, the feasible solution set is filtered by engineering constraints. In this step, unreasonable solutions are removed. For example, to ensure that the solutions are conforming to the definition of pipe grading, the overlapped length of the adjacent subpipe grades must be larger than zero. In addition, to ensure the installation requirements of pipe fittings and components, the minimum distance of adjacent bends/T-junctions should be kept. Finally, the fitness of each subpipe grade of the solution is calculated by (7) and (8), and all solutions are sorted by 


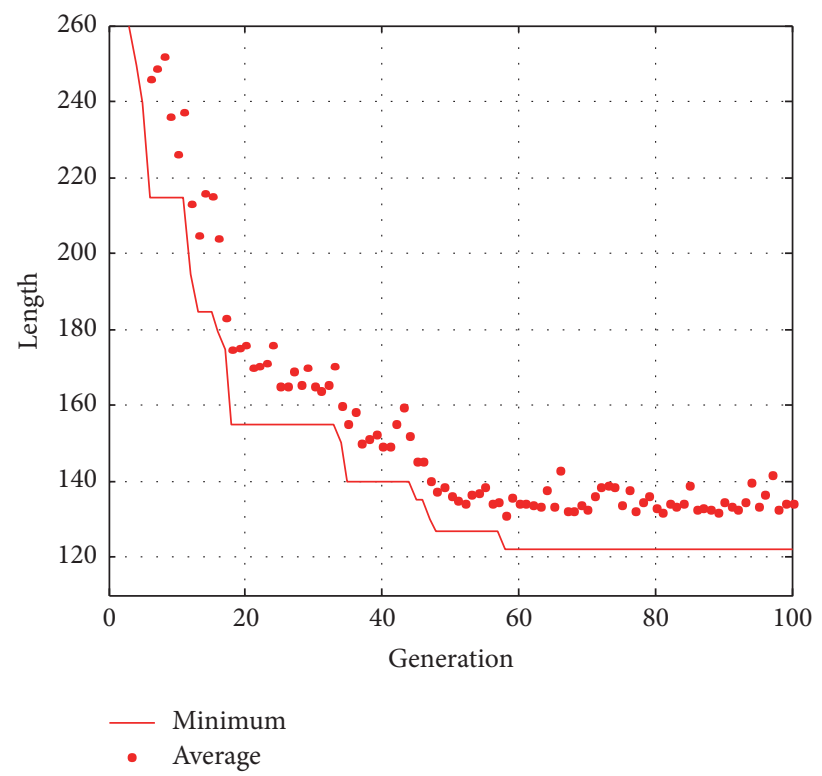

(a)

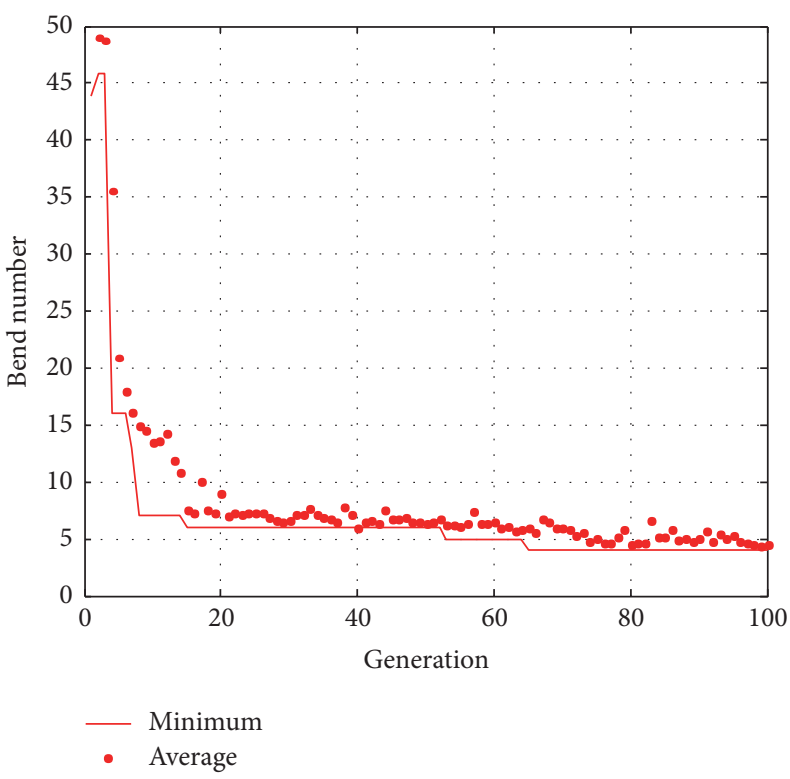

(b)

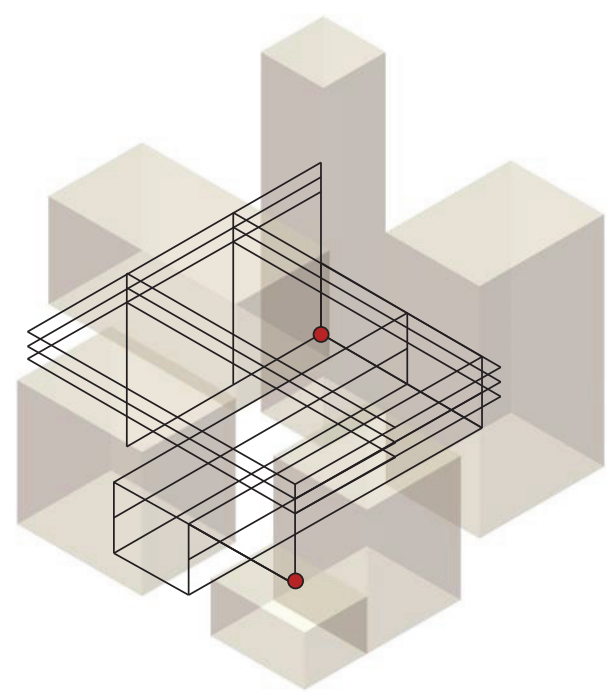

(c)

FIGURE 18: Evolutionary graphs of pipe grade 2. (a) Evolution process of length; (b) evolution process of bend numbers; (c) Pareto optimal set.

$\mu^{k}$. Solution with largest $\mu^{k}$ is chosen as the best compromise solution.

Some best solutions are listed in Table 3. As shown in Table 3, solution 1 is selected as the best compromise solution. The routing result of the branch pipeline is shown in Figure 20.

As shown in Figures 17-19, the individuals of each subpipe grade in the design example converge to the some appropriate route after around 60 th generation. The same branch pipeline example executed by a modified geneticalgorithm-based approach proposed in literature [40] shows that the individuals converge to near-optimal solution after 21 generations. According to the simulation test result, the convergence speed of the proposed method is relatively slow compared with that of literature [40]. For the best compromise solution obtained by the proposed method, the total length is 197 and the number of bends is $8(2 \mathrm{~T}$ junctions included), which is same with the near-optimal solution obtained by the literature [40]. But the merit of proposed approach lies in that it could acquire more feasible solutions that will provide the designer with more reliable references. In addition, the proposed method provides a new way for routing pipelines with different pipe diameters based on concept of pipe grading.

4.2. Case Study 2: A Fuel Piping System Design of Ship Engine Room. Figure 21 shows the schematic diagram of a fuel piping system in a ship engine room. The main equipment 


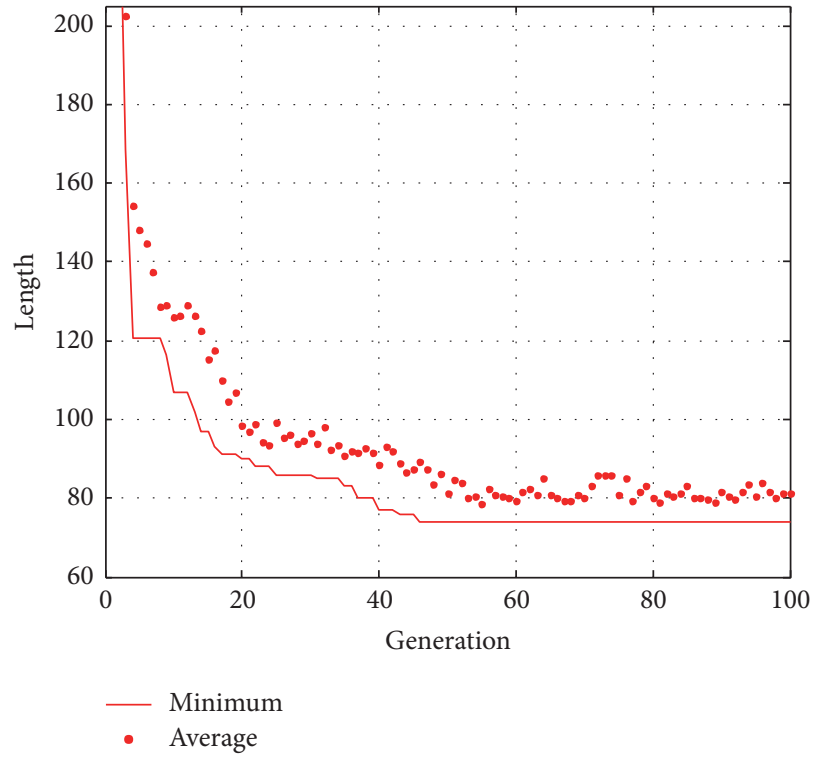

(a)

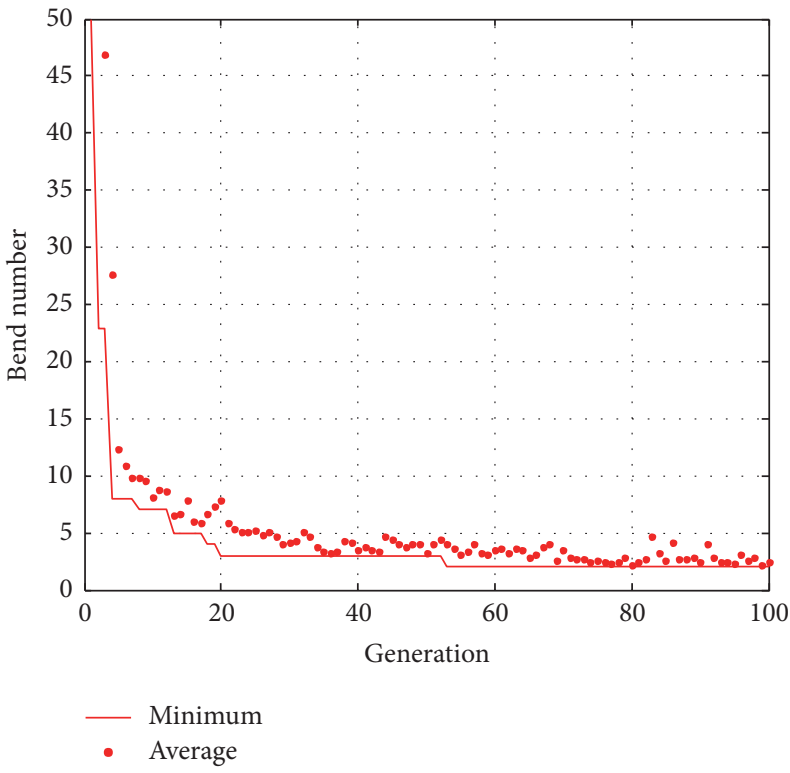

(b)

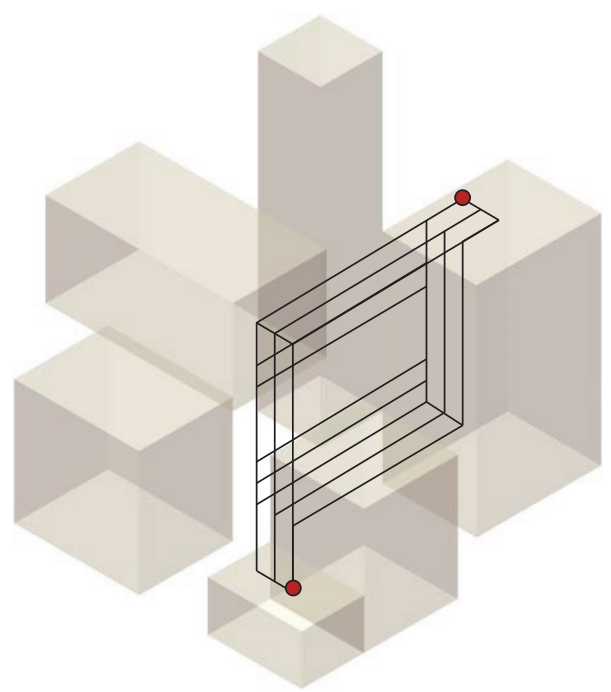

(c)

Figure 19: Evolutionary graphs of pipe grade 2. (a) Evolution process of length; (b) evolution process of bend numbers; (c) Pareto optimal set.

TABLE 3: Some solutions of test example.

\begin{tabular}{|c|c|c|c|c|c|c|c|c|c|c|c|c|}
\hline Solution & $L_{P}$ & $B_{P}$ & $L_{P_{1}}$ & $B_{P_{1}}$ & $L_{P_{2}}$ & $B_{P_{2}}$ & $L_{P_{3}}$ & $B_{P_{3}}$ & $O_{P_{1}-P_{2}}$ & $\mathrm{O}_{P_{1}-P_{3}}$ & $\mathrm{O}_{P_{2}-P_{3}}$ & $\mu^{k}$ \\
\hline 1 & 197 & 8 & 94 & 3 & 118 & 5 & 74 & 4 & 50 & - & 39 & 0.093095 \\
\hline 2 & 197 & 8 & 94 & 3 & 120 & 5 & 74 & 4 & 51 & - & 40 & 0.091905 \\
\hline 3 & 205 & 8 & 94 & 3 & 128 & 4 & 74 & 4 & 43 & - & 40 & 0.090952 \\
\hline 4 & 197 & 8 & 94 & 4 & 120 & 4 & 74 & 4 & 51 & - & 40 & 0.085119 \\
\hline 5 & 197 & 8 & 94 & 3 & 140 & 5 & 74 & 4 & 61 & - & 50 & 0.08 \\
\hline 6 & 197 & 8 & 94 & 4 & 132 & 4 & 74 & 4 & 58 & - & 45 & 0.077976 \\
\hline 7 & 205 & 8 & 94 & 3 & 140 & 5 & 74 & 4 & 53 & - & 50 & 0.075119 \\
\hline 8 & 197 & 8 & 94 & 4 & 140 & 4 & 74 & 4 & 61 & - & 50 & 0.073214 \\
\hline 9 & 197 & 8 & 94 & 3 & 152 & 5 & 74 & 4 & 67 & - & 56 & 0.072857 \\
\hline 10 & 197 & 8 & 94 & 3 & 156 & 5 & 74 & 4 & 69 & - & 58 & 0.070476 \\
\hline
\end{tabular}


TABLE 4: Diagonal coordinates of main parts of the simplified models.

\begin{tabular}{|c|c|c|}
\hline Equipment name & Diagonal coordinate 1 & Diagonal coordinate 2 \\
\hline Fuel oil storage tank 1 & $(1,67,23)$ & $(40,107,103)$ \\
\hline Fuel oil storage tank 2 & $(1,67,183)$ & $(40,107,263)$ \\
\hline \multirow{2}{*}{ Steam boiler } & $(90,1,290)$ & $(115,17,311)$ \\
\hline & $(115,1,283)$ & $(152,83,320)$ \\
\hline \multirow{2}{*}{ Hot water boiler } & $(135,15,1)$ & $(152,55,17)$ \\
\hline & $(152,19,5)$ & $(163,29,13)$ \\
\hline \multirow{4}{*}{ Diesel generator 1} & $(122,15,97)$ & $(198,17,127)$ \\
\hline & $(123,17,104)$ & $(139,41,116)$ \\
\hline & $(128,17,104)$ & $(46,33,118)$ \\
\hline & $(160,17,103)$ & $(188,42,117)$ \\
\hline \multirow{4}{*}{ Diesel generator 2} & $(122,15,163)$ & $(198,23,188)$ \\
\hline & $(123,17,170)$ & $(139,41,181)$ \\
\hline & $(138,17,170)$ & $(157,33,183)$ \\
\hline & $(160,17,169)$ & $(188,42,183)$ \\
\hline \multirow{4}{*}{ Marine main engine 1} & $(110,11,42)$ & $(122,35,65)$ \\
\hline & $(146,9,47)$ & $(175,15,60)$ \\
\hline & $(136,15,47)$ & $(187,46,66)$ \\
\hline & $(122,42,40)$ & $(196,54,55)$ \\
\hline \multirow{4}{*}{ Marine main engine 2} & $(110,11,222)$ & $(122,35,246)$ \\
\hline & $(146,9,228)$ & $(175,15,241)$ \\
\hline & $(136,15,228)$ & $(187,46,247)$ \\
\hline & $(122,42,220)$ & $(196,54,245)$ \\
\hline Fuel transfer pump 1 & $(17,47,105)$ & $(33,56,111)$ \\
\hline Fuel transfer pump 2 & $(41,45,105)$ & $(57,53,111)$ \\
\hline
\end{tabular}

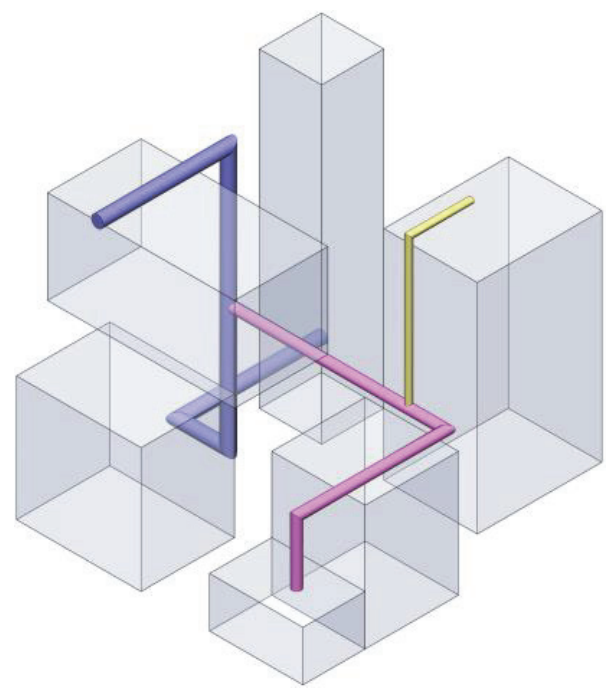

FIGURE 20: The routing result of solution 1.

involved in the schematic diagram includes fuel oil tank, fuel oil storage tank, fuel transfer pump, steam boiler, hot water boiler, boiler burner, marine main engine, and diesel generator. Relationships between connected structure and equipment are represented by six lines of different colors.

By using 3D CAD software SolidWorks, the solid model of related equipment is established by the method presented in Section 2.1.1, which is assembled according to the relationships of their actual installation locations. The pipe routing space is set as $5700 \mathrm{~mm} \times 3360 \mathrm{~mm} \times 960 \mathrm{~mm}$, which is determined by the occupied spaces of equipment and the concept of adaptive region introduced in Section 3.2. In accordance with the minimum value of the pipe diameter, the size of the cube grid is set as $30 \mathrm{~mm} \times 30 \mathrm{~mm} \times 30 \mathrm{~mm}$. Therefore, the working space is then divided into $190 \times 112 \times$ 320 uniform 3D cubic grid cells. Table 4 shows the diagonal coordinates of the main parts of the simplified models.

According to the schematic diagram, the fuel piping system is classified into six pipelines. The coordinates, representing the locations of connecting points and the diameter parameters of pipes which the connecting point belongs to, are summarized in Table 5. Seamless steel tubes are used in fuel piping system, and the outer diameter and wall thickness of pipe are represented by the diameter parameters. By taking $\phi 48 \times 4$ as an example, $48 \mathrm{~mm}$ and $4 \mathrm{~mm}$ are, respectively, outer diameter and wall thickness of a pipe. In addition, to eliminate the influence of the directionality of connecting point on the pipe route planning, the location of 
TABLE 5: Information of piping route path and connecting points.

Route path name Included cabins and equipment with corresponding coordinates of connecting points Fuel oil tank: $(58,21,6),(58,21,304)$ Pipe specification $(\mathrm{mm})$

Path 1

Fuel transfer pump 1: $(39,49,106)$

$\phi 48 \times 4$

Fuel transfer pump 2: $(15,52,106)$

$\phi 48 \times 4$

Fuel transfer pump 1: $(39,49,113)$

$\phi 48 \times 4$

Fuel transfer pump 2: $(15,52,113)$

Fuel oil storage tank 1: $(33,70,104)$

$\phi 48 \times 4$

Path 2

Fuel oil storage tank 2: $(33,70,182)$

$\phi 48 \times 4$

$\phi 48 \times 4$

$\phi 48 \times 4$

Fuel oil storage tank 1: $(20,61,69)$

$\phi 34 \times 3.5$

Fuel oil storage tank 2: $(20,61,216)$

$\phi 34 \times 3.5$

Path 3

Diesel generator 1: $(181,33,122),(185,33,122)$

$\phi 22 \times 2$

Diesel generator 2: $(181,33,188),(185,33,188)$

$\phi 22 \times 2$

Fuel oil storage tank 1: $(20,61,63)$

Fuel oil storage tank 2: $(20,61,223)$

$\phi 42 \times 3.5$

$\phi 42 \times 3.5$

Path 4

Marine main engine $1:(139,40,67),(144,40,67)$

$\phi 34 \times 2.5$

Marine main engine 2: $(139,40,248),(144,40,248)$

$\phi 34 \times 2.5$

Fuel oil storage tank 1: $(20,61,56)$

Fuel oil storage tank 2: $(20,61,229)$

Hot water boiler: $(159,23,14)$

$\phi 42 \times 3.5$

$\phi 42 \times 3.5$

Path 5

Steam boiler: $(101,13,289)$

$\phi 34 \times 2.5$

$\phi 34 \times 2.5$

Fuel oil storage tank 1: $(20,61,31)$

Fuel oil storage tank 2: $(20,61,254)$

$\phi 22 \times 2$

$\phi 22 \times 2$

$\phi 22 \times 2$

Hot water boiler: $(161,21,14)$

$\phi 22 \times 2$

Steam boiler: $(96,5,289)$

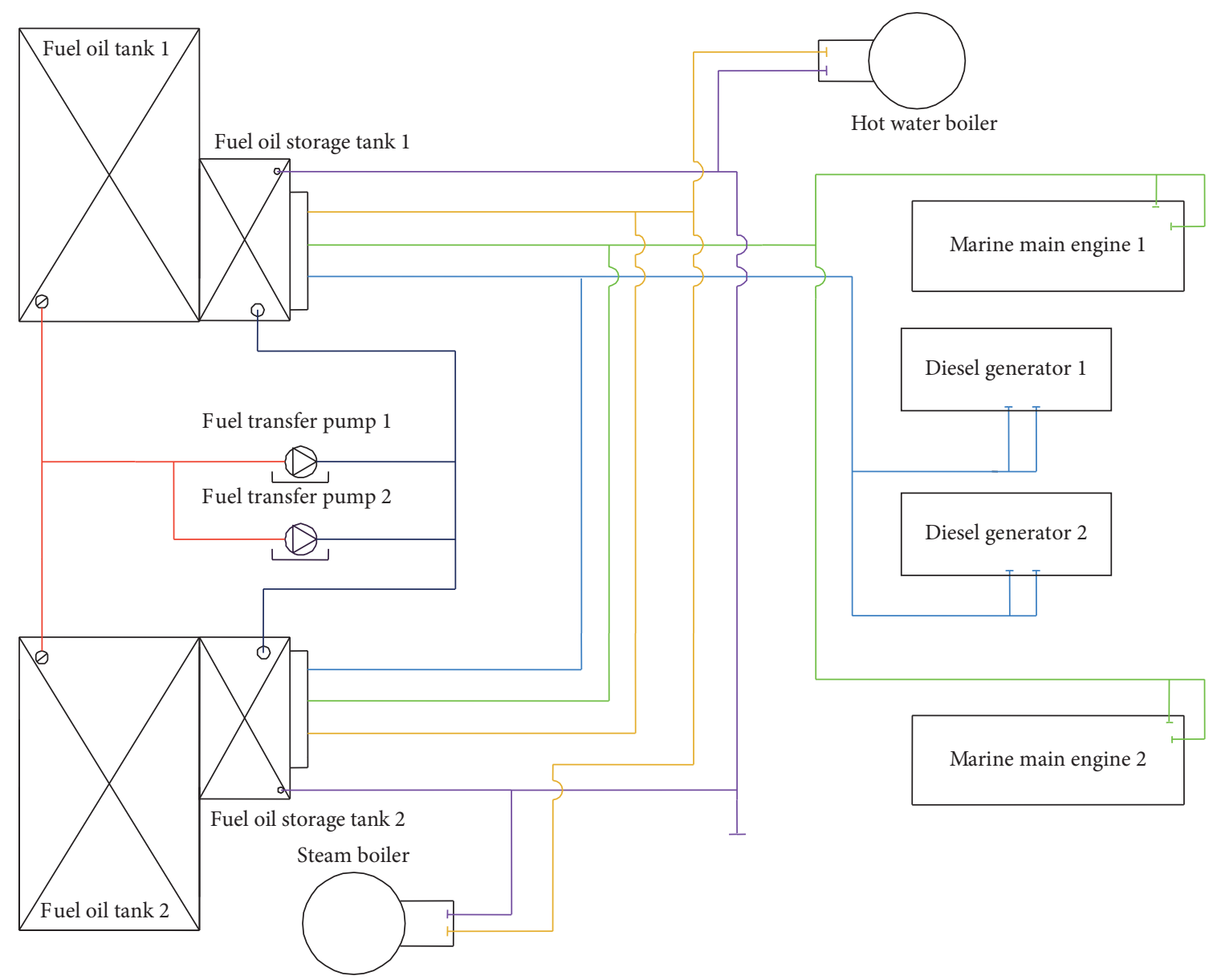

FIgURE 21: A schematic diagram of the fuel piping system in a ship engine room. 


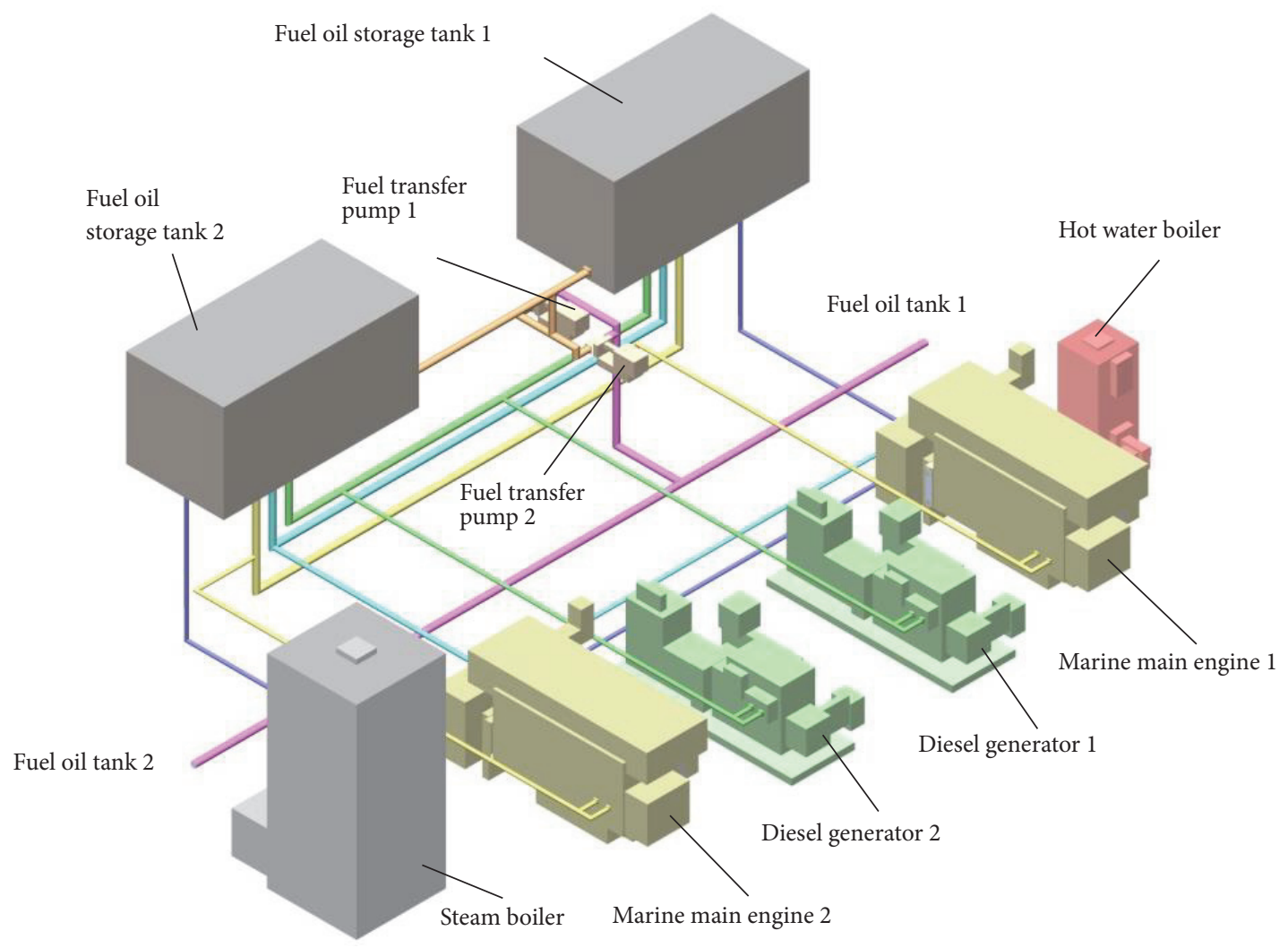

FIGURE 22: Final routing result of the fuel pipe system in a ship engine room.

TABLE 6: Genetic algorithm parameters.

\begin{tabular}{lcc}
\hline & MA-NSGA-II & MA-CCNSGA-II \\
\hline Population size & 30 & 40 \\
Number of generation & 100 & 200 \\
Crossover probability & 0.8 & 0.8 \\
Mutation probability & 0.05 & 0.05 \\
\hline
\end{tabular}

the connection point is extended properly along the specific direction during the routing progress, which also makes the pipe routing design more flexible and the piping layout results more reasonable.

Based on the established space model, the fuel piping system could be designed by using the algorithm proposed in Section 3. By considering the maximum diameter value of the pipelines, the planning order of the six pipelines is as follows: pipe 1 or pipe 2, pipe 4 or pipe 5, pipe 3 , and pipe 6 .

Pipe path 3 is taken as an example to illustrate the algorithm procedure of pipe route planning. According to the concept of pipe grading defined in Section 2.2.3, the connecting points between two fuel oil storage tanks, the coordinates of which are $(20,61,69)$ and $(20,61,216)$, are defined as points-grade 1, which will be connected preferentially; the connecting points of diesel generator, the coordinates of which are $(180,30,122),(185,30,122),(181$, $30,188)$ and $(185,30,188)$, are defined as points-grade 2 , which will be connected after the routing of pipe grade 1 . In this example, as pipe grade 1 includes two connecting points,
MA-NSGA-II is adopted as the pipe routing algorithm. For pipe grade 2, four connecting points are contained, and MACCNSGA-II is applied for pipe route planning. Based on the concept of key connecting point given in Section 3.1, the point $(20,61,216)$ is selected as the start point that will connect the points of pipe grade 2 sequentially. Accordingly, four groups of initial individuals are generated, and the best individual for each population is selected by fuzzy set theory, which is repeated during the optimization process. The parameters of the optimization algorithm are shown in Table 6. Optimal solution set construction method and the compromise solution selected method are similar as illustrated in Section 4.1.

According to the results obtained by using the proposed algorithm, the parametric CAD Model of the fuel pipe system is established by utilizing the SolidWorks API and VB.NET. The routing results of the pipelines are summarized in Table 7. The CAD Model of fuel pipe system is shown in Figure 22. The optimized result by the proposed algorithm could provide a better reference for the designers. Generally, 
TABLE 7: Route paths of fuel path system.

\begin{tabular}{|c|c|c|c|}
\hline \multirow{2}{*}{ Route path name } & \multirow{2}{*}{ 3D solid model of pipe } & \multicolumn{2}{|c|}{ Parameters } \\
\hline & & Grade 1 & Grade 2 \\
\hline Path 1 & & $\begin{array}{c}L_{P}=403 \\
B_{P}=5 \\
D_{P}=48 \mathrm{~mm}\end{array}$ & - \\
\hline Path 2 & & $\begin{array}{c}L_{P}=133 \\
B_{P}=5 \\
D_{P}=48 \mathrm{~mm}\end{array}$ & - \\
\hline Path 3 & & $\begin{array}{c}L_{P}=209 \\
B_{P}=2 \\
D_{P}=34 \mathrm{~mm}\end{array}$ & $\begin{array}{c}L_{P}=469 \\
B_{P}=7 \\
O_{P}=117 \\
D_{P}=22 \mathrm{~mm}\end{array}$ \\
\hline Path 4 & & $\begin{array}{c}L_{P}=269 \\
B_{P}=2 \\
D_{P}=42 \mathrm{~mm}\end{array}$ & $\begin{array}{c}L_{P}=792 \\
B_{P}=9 \\
O_{P}=204 \\
D_{P}=34 \mathrm{~mm}\end{array}$ \\
\hline Path 5 & & $\begin{array}{c}L_{P}=233 \\
B_{P}=2 \\
D_{P}=42 \mathrm{~mm}\end{array}$ & $\begin{array}{c}L_{P}=526 \\
B_{P}=6 \\
O_{P}=107 \\
D_{P}=34 \mathrm{~mm}\end{array}$ \\
\hline Path 6 & & $\begin{array}{c}L_{P}=618 \\
B_{P}=7 \\
D_{P}=22 \mathrm{~mm}\end{array}$ & - \\
\hline
\end{tabular}

minor modification is enough for actual application, which demonstrates the effectiveness of the proposed methodology and the important guiding significance to actual piping system design.

\section{Conclusions}

In order to improve design efficiency and to reduce human errors, an optimized design method for branch pipe routing is proposed in this paper. To cope with the diameter differences of branch pipelines, the concept of pipe grading is proposed in this paper. In consideration of the number differences of connecting points in each grade, a pipe routing optimization procedure is proposed by combining MA, NSGA-II, and CCNSGA-II. Based on the concept of pipe grading, the requirement of usage safety can be considered at initial design stage, which lay a solid foundation for detailed design. To improve the performance of genetic algorithm procedure, fixed-length encoding method is employed in our proposed design optimization procedure. By utilizing the proposed pipe routing optimization procedure, optimal solution set of branch pipe is obtained; then the best compromise solution can be selected by taking full account of the important indicators of pipeline, such as bending number, subpipe length, and overlapped length as well as other complicated engineering constraints.

A design example of branch pipeline is conducted and design optimization of a fuel piping system in an actual ship is implemented to verify the feasibility and effectiveness of the proposed methodology. Further work will be carried out to develop a comprehensive and user-friendly computeraided pipe routing system, which employs the proposed pipe routing optimization method.

\section{Competing Interests}

The authors declare that they have no competing interests.

\section{Acknowledgments}

This work is funded by the National Natural Science Foundation of China (Grant no. 51275340).

\section{References}

[1] E. W. Dijkstra, "A note on two problems in connexion with graphs," Numerische Mathematik, vol. 1, no. 1, pp. 269-271, 1959. 
[2] P. E. Hart, N. J. Nilsson, and B. Raphael, "A formal basis for the heuristic determination of minimum cost paths," IEEE Transactions on Systems Science \& Cybernetics, vol. 4, no. 2, pp. 100-107, 1968.

[3] C. Y. Lee, "An algorithm for path connections and its applications," IRE Transactions on Electronic Computers, vol. 10, no. 3 , pp. 346-365, 1961.

[4] D. W. Hightower, "A solution to line routing problems on the continuous plane," in Proceedings of the 25 Years of DAC Papers on Twenty-Five Years of Electronic Design Automation, pp. 11-34, ACM, 1988.

[5] P. W. Rourke, Development of a three-dimensional pipe routing algorithm [Ph.D. thesis], Lehigh University, Bethlehem, $\mathrm{Pa}$, USA, 1975.

[6] T. Mitsuta, Y. Kobayashi, Y. Wada et al., "A knowledge-based approach to routing problems in industrial plant design," in Proceedings of the 6th Internationl Workshop Vol. 1 on Expert Systems \& Their Applications, pp. 237-256, Avignon, France, 1987.

[7] J. Fan, M. Ma, and X. G. Yang, "Research on automatic laying out for external pipeline of aero-engine," Journal of Machine Design, vol. 20, no. 7, pp. 21-23, 2003.

[8] C. E. Wang and Q. Liu, "Projection and geodesic-based pipe routing algorithm," IEEE Transactions on Automation Science and Engineering, vol. 8, no. 3, pp. 641-645, 2011.

[9] J. H. Holland, Adaptation in Natural and Artificial Systems, University of Michigan Press, Ann Arbor, Mich, USA, 1975.

[10] T. Ito, "Genetic algorithm approach to piping route path planning," Journal of Intelligent Manufacturing, vol. 10, no. 1, pp. 103-114, 1999.

[11] T. Ito, "Heuristics for route generation in pipe route planning," in Proceedings of the 12th European Simulation Symposium (ESS '00), pp. 178-182, Hamburg, Germany, 2000.

[12] T. Ito, "Route planning wizard: basic concept and its implementation," Lecture Notes in Computer Science (including subseries Lecture Notes in Artificial Intelligence and Lecture Notes in Bioinformatics), vol. 2358, pp. 547-556, 2002.

[13] X. N. Fan, Y. Lin, and Z. H. Ji, "A variable length coding genetic algorithm to ship pipe path routing optimization in 3D space," Shipbuilding of China, vol. 48, no. 1, pp. 82-90, 2007.

[14] S. Sandurkar and W. Chen, "GAPRUS-genetic algorithms based pipe routing using tessellated objects," Computers in Industry, vol. 38, no. 3, pp. 209-223, 1999.

[15] H. L. Wang, C. L. Zhao, W. Ch. Yan et al., "Three-dimensional multi-pipe route optimization based on genetic algorithms," in Knowledge Enterprise: Intelligent Strategies in Product Design, Manufacturing, and Management, Proceedings of PROLAMAT 2006, IFIP TC5 International Conference, Shanghai, China, 2006, pp. 177-183, Springer, 2006.

[16] Y. Kanemoto, R. Sugawara, and M. Ohmura, "A genetic algorithm for the rectilinear steiner tree in 3-D VLSI layout design," in Proceedings of the 47th Midwest Symposium on Circuits and Systems (MWSCAS '04), pp. I-465-I-468, Hiroshima, Japan, July 2004.

[17] T. Ren, Z.-L. Zhu, G. M. Dimirovski, Z.-H. Gao, X.-H. Sun, and $\mathrm{H}$. Yu, "A new pipe routing method for aero-engines based on genetic algorithm," Proceedings of the Institution of Mechanical Engineers, Part G: Journal of Aerospace Engineering, vol. 228, no. 3, pp. 424-434, 2014.

[18] A. Colorni, M. Dorigo, V. Maniezzo et al., "Distributed optimization by ant colonies," in Proceedings of the European
Conference on Artificial Life (ECAL '91), pp. 134-142, Paris, France, 1991.

[19] M. Dorigo and L. M. Gambardella, "Ant colony system: a cooperative learning approach to the traveling salesman problem," IEEE Transactions on Evolutionary Computation, vol. 1, no. 1, pp. 53-66, 1997.

[20] X. N. Fan, Y. Lin, and Z. H. Ji, "Ship pipe routing design using the ACO with iterative pheromone updating," Journal of Ship Production, vol. 23, no. 1, pp. 36-45, 2007.

[21] X.-N. Fan, Y. Lin, and Z.-H. Ji, "Multi ant colony cooperative coevolution for optimization of ship multi pipe parallel routing," Journal of Shanghai Jiaotong University, vol. 43, no. 2, pp. $193-$ 197, 2009 (Chinese).

[22] W.-Y. Jiang, Y. Lin, M. Chen, and Y.-Y. Yu, "A co-evolutionary improved multi-ant colony optimization for ship multiple and branch pipe route design," Ocean Engineering, vol. 102, pp. 6370, 2015.

[23] Y. F. Qu, D. Jiang, G. Y. Gao, and Y. Huo, "Pipe routing approach for aircraft engines based on ant colony optimization," Journal of Aerospace Engineering, vol. 29, no. 3, 2016.

[24] W.-Y. Jiang, Y. Lin, M. Chen, and Y.-Y. Yu, "An ant colony optimization-genetic algorithm approach for ship pipe route design," International Shipbuilding Progress, vol. 61, no. 3-4, pp. 163-183, 2014.

[25] J. Kennedy, "Particle swarm optimization," in Encyclopedia of Machine Learning, pp. 760-766, Springer US, 2010.

[26] A. Asmara and U. Nienhuis, "Automatic piping system in ship," in Proceedings of the 5th International Conference on Computer and IT Applications in the Maritime Industries, Sieca Repro (TUD '06), pp. 269-280, Delft, The Netherlands, 2006.

[27] Q. Liu and C. E. Wang, "A discrete particle swarm optimization algorithm for rectilinear branch pipe routing," Assembly Automation, vol. 31, no. 4, pp. 363-368, 2011.

[28] D. Vakil and M. R. Zargham, "An expert system for channel routing," in Proceedings of the 1st International Conference on Industrial and Engineering Applications of Artificial Intelligence and Expert Systems (IEA/AIE '88), vol. 2, pp. 1033-1039, Tullahoma, Tenn, USA, June 1988.

[29] J.-H. Park and R. L. Storch, "Pipe-routing algorithm development: case study of a ship engine room design," Expert Systems with Applications, vol. 23, no. 3, pp. 299-309, 2002.

[30] E. E. S. Calixto, P. G. Bordeira, H. T. Calazans, C. A. C. Tavares, and M. T. D. Rodriguez, "Plant design project automation using an automatic pipe routing routine," Computer Aided Chemical Engineering, vol. 27, pp. 807-812, 2009.

[31] X.-Y. Shao, X.-Z. Chu, H.-B. Qiu, L. Gao, and J. Yan, "An expert system using rough sets theory for aided conceptual design of ship's engine room automation," Expert Systems with Applications, vol. 36, no. 2, pp. 3223-3233, 2009.

[32] J. Wu, Y. Lin, Z. S. Ji et al., "Optimal approach of ship branch pipe routing optimization based on co-evolutionary algorithm," Ship and Ocean Engineering, vol. 37, no. 4, pp. 135-138, 2008.

[33] Q. Liu and C. Wang, "Multi-terminal pipe routing by Steiner minimal tree and particle swarm optimisation," Enterprise Information Systems, vol. 6, no. 3, pp. 315-327, 2012.

[34] L. Q. Wang and Q. L. He, "Reciprocating compressor pipeline vibration analysis and engineering application," Fluid Machinery, vol. 30, no. 10, pp. 28-31, 2002.

[35] K. Deb, A. Pratap, S. Agarwal, and T. Meyarivan, "A fast and elitist multiobjective genetic algorithm: NSGA-II," IEEE Transactions on Evolutionary Computation, vol. 6, no. 2, pp. 182197, 2002. 
[36] B. Dorronsoro, G. Danoy, A. J. Nebro, and P. Bouvry, "Achieving super-linear performance in parallel multi-objective evolutionary algorithms by means of cooperative coevolution," Computers \& Operations Research, vol. 40, no. 6, pp. 1552-1563, 2013.

[37] G. Theodosiou and N. S. Sapidis, "Information models of layout constraints for product life-cycle management: a solidmodelling approach," CAD Computer Aided Design, vol. 36, no. 6, pp. 549-564, 2004.

[38] A. Asmara, U. Nienhuis, and R. Hekkenberg, "Approximate orthogonal simplification of $3 \mathrm{D}$ model ", in Proceedings of the IEEE World Congress on Computational Intelligence (WCCI '10)_IEEE Congress on Evolutionary Computation (CEC '10), July 2010.

[39] M. A. Abido, "Multiobjective evolutionary algorithms for electric power dispatch problem," IEEE Transactions on Evolutionary Computation, vol. 10, no. 3, pp. 315-329, 2006.

[40] H. T. Sui and W. T. Niu, "Branch-pipe-routing approach for ships using improved genetic algorithm," Frontiers of Mechanical Engineering, vol. 11, no. 3, pp. 316-323, 2016. 


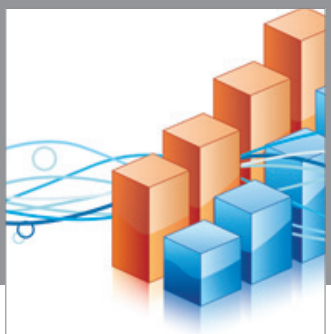

Advances in

Operations Research

vatem alat4

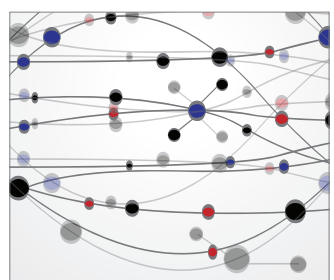

\section{The Scientific} World Journal
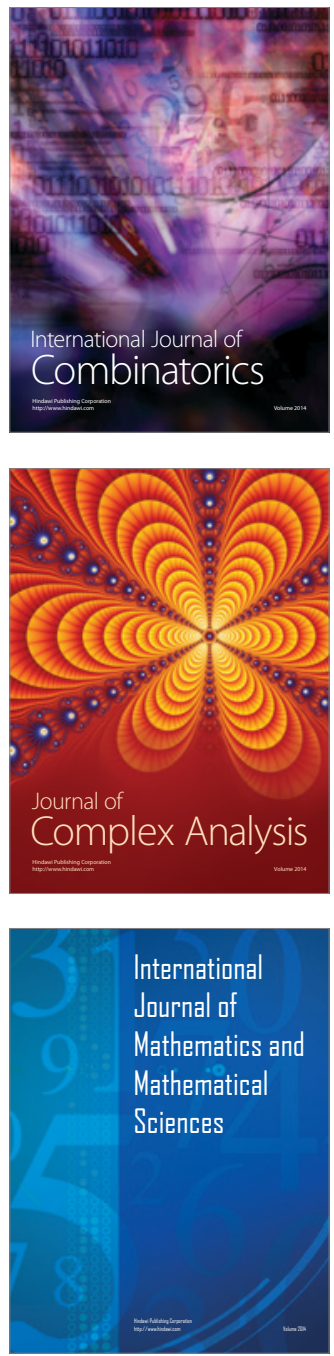
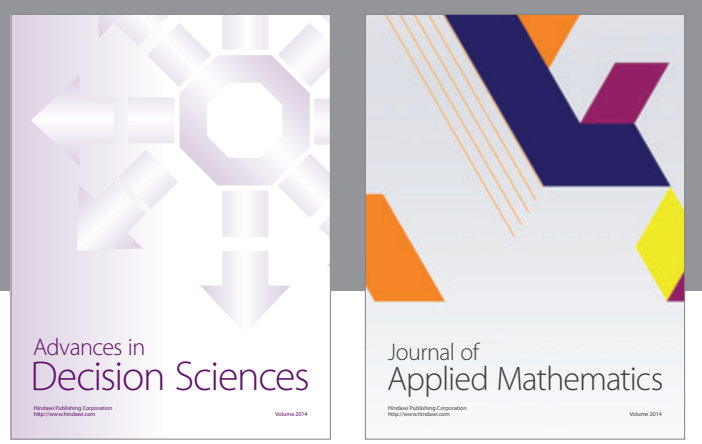

Algebra

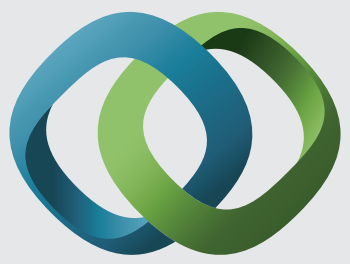

\section{Hindawi}

Submit your manuscripts at

http://www.hindawi.com
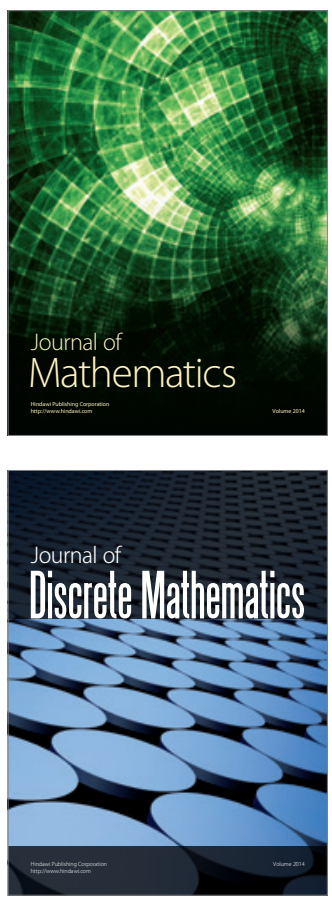

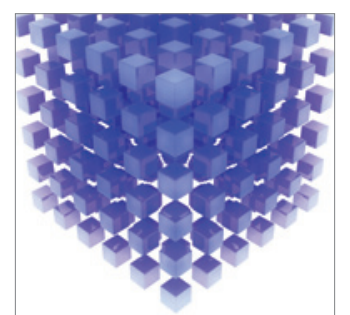

Mathematical Problems in Engineering
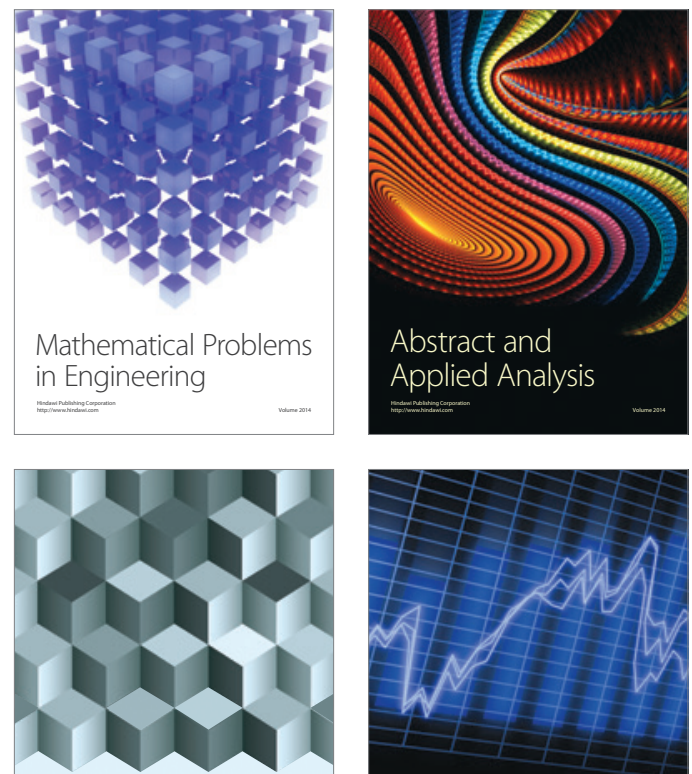

Journal of

Function Spaces

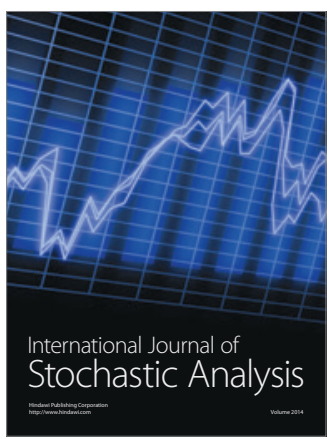

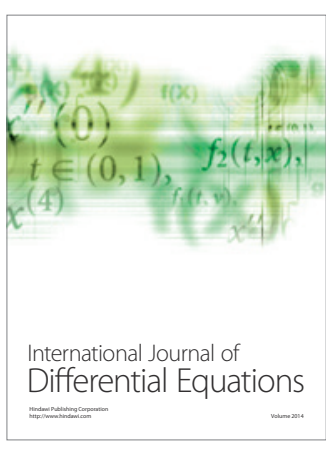
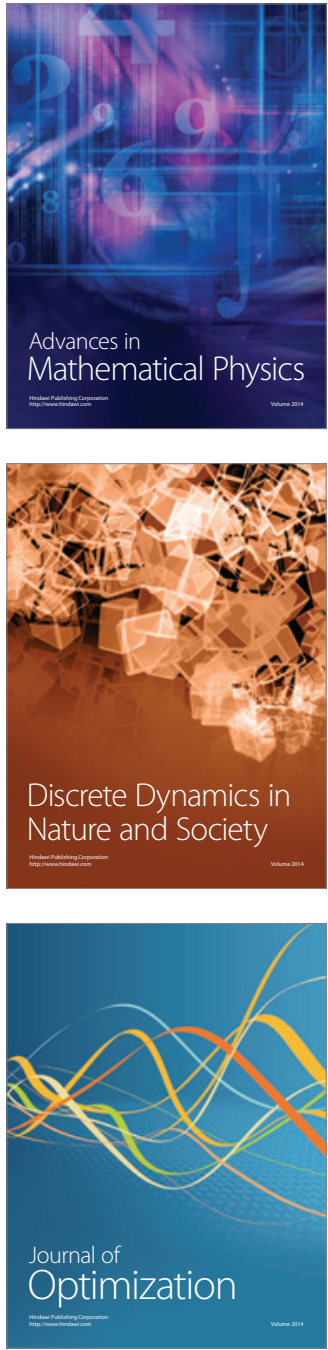